\title{
Relaxation of some multi-well problems
}

\author{
Kaushik Bhattacharya \\ Division of Engineering and Applied Science, \\ California Institute of Technology, Pasadena, \\ CA 91125, USA (bhatta@caltech.edu)
}

\section{Georg Dolzmann}

Department of Mathematics, University of Maryland, College Park, MD 20742, USA (dolzmann@math.umd.edu)

(MS received 21 October 1998; accepted 11 May 1999)

\begin{abstract}
Mathematical models of phase transitions in solids lead to the variational problem, minimize $\int_{\Omega} W(D u) \mathrm{d} x$, where $W$ has a multi-well structure, i.e. $W=0$ on a multi-well set $K$ and $W>0$ otherwise. We study this problem in two dimensions in the case of equal determinant, i.e. for $K=\mathrm{SO}(2) U_{1} \cup \cdots \cup \mathrm{SO}(2) U_{k}$ or $K=\mathrm{O}(2) U_{1} \cup \cdots \cup \mathrm{O}(2) U_{k}$ for $U_{1}, \ldots, U_{k} \in \mathbb{M}^{2 \times 2}$ with $\operatorname{det} U_{i}=\delta$, in three dimensions when the matrices $U_{i}$ are essentially two-dimensional and also for $K=\operatorname{SO}(3) \hat{U}_{1} \cup \cdots \cup \operatorname{SO}(3) \hat{U}_{k}$ for $U_{1}, \ldots, U_{k} \in \mathbb{M}^{3 \times 3}$ with $\left(\operatorname{adj} U_{i}^{\mathrm{T}} U_{i}\right)_{33}=\delta^{2}$, which arises in the study of thin films. Here, $\hat{U}_{i}$ denotes the $(3 \times 2)$ matrix formed with the first two columns of $U_{i}$. We characterize generalized convex hulls, including the quasiconvex hull, of these sets, prove existence of minimizers and identify conditions for the uniqueness of the minimizing Young measure. Finally, we use the characterization of the quasiconvex hull to propose 'approximate relaxed energies', quasiconvex functions which vanish on the quasiconvex hull of $K$ and grow quadratically away from it.
\end{abstract}

\section{Introduction}

Mathematical models for phase transitions in solids lead to the following variational problem (see $[2,4])$. Minimize

$$
I(u)=\int_{\Omega} W(D u) \mathrm{d} x,
$$

where $u: \Omega \subset \mathbb{R}^{n} \rightarrow \mathbb{R}^{n}$ is the deformation of an elastic body which occupies in an ideal unstressed configuration the domain $\Omega$. We assume that the stored energy density $W$ is non-negative and that the level set $K=\{W=0\}$ is not empty. The principle of material frame indifference and symmetry properties of the underlying material imply further structure of $K$. For many materials of interest, $K$ has a multi-well structure,

$$
K=\bigcup_{i=1}^{k} \operatorname{SO}(n) U_{i} .
$$

As a consequence, $W$ fails to be quasiconvex and therefore the existence of minimizers cannot be obtained from the direct method in the calculus of variations based 
on sequential lower semicontinuity of the integral. However, the behaviour of the minimization problem is closely related to the quasiconvex hull $K^{\mathrm{qc}}$ of the set $K$ : if we minimize $I$ on all Sobolev functions which coincide with the affine mapping $u(x)=F x$ on $\partial \Omega$, then the infimum of $I$ is zero if and only if $F$ belongs to the quasiconvex hull $K^{\mathrm{qc}}$ of $K$ (see [19]).

In this paper we characterize generalized convex hulls for multi-well problems in two and three dimensions in the case of equal determinant, i.e. when $\operatorname{det} U_{i}=\delta$. In this case, $K^{\mathrm{qc}}$ is contained in the set $\{\operatorname{det} F=\delta\}$, which in two dimensions has the remarkable property that any two points $F_{1}, F_{2}$ are rank-one connected in the following sense: there exists a $Q \in \mathrm{SO}(2)$ such that the rank $\left(Q F_{2}-F_{2}\right)$ is equal to one (see [8] or lemma 2.2 below).

We prove the following results (see $\S 2$ below for the notation used).

Theorem 1.1. Let $\mathcal{U}=\left\{U_{1}, \ldots, U_{k}\right\} \subset \mathbb{M}_{\text {sym }}^{2 \times 2}$, where the matrices $U_{i}$ are positivedefinite and satisfy $\operatorname{det} U_{i}=\delta>0$.

(i) Let $K=\mathrm{SO}(2) U_{1} \cup \cdots \cup \mathrm{SO}(2) U_{k}$. Then $K^{(2)}=K^{\mathrm{lc}}=K^{\mathrm{rc}}=K^{\mathrm{qc}}=K^{\mathrm{pc}}$. Further, if

$$
\left\{U_{1}, \ldots, U_{n}\right\}=\left\{U_{i} \in \mathcal{U}:\left|U_{i} \tilde{e}\right|^{2}>\max _{j \neq i}\left|U_{j} \tilde{e}\right|^{2} \quad \text { for some } \tilde{e} \in S^{1}\right\},
$$

then there exists a set $\mathcal{E}_{n}=\left\{e_{1}, \ldots, e_{n}\right\} \subset S^{1}$ such that any of these hulls is given by

$$
\left\{F \in \mathbb{M}^{2 \times 2}: \operatorname{det} F=\delta,\left|F e_{i}\right|^{2} \leqslant \max _{j=1, \ldots, n}\left|U_{j} e_{i}\right|^{2}, i=1, \ldots, n\right\} .
$$

(ii) Let $K=\mathrm{O}(2) U_{1} \cup \cdots \cup \mathrm{O}(2) U_{k}$. Then $K^{(3)}=K^{\mathrm{lc}}=K^{\mathrm{rc}}=K^{\mathrm{qc}}=K^{\mathrm{pc}}$ and any of these hulls is given by

$$
\left\{F \in \mathbb{M}^{2 \times 2}:|\operatorname{det} F| \leqslant \delta,|F e|^{2} \leqslant \max _{i=1, \ldots, k}\left|U_{i} e\right|^{2} \forall e \in S^{1}\right\} .
$$

A similar result holds for the three-dimensional case if the wells are essentially two dimensional.

Theorem 1.2. Let $\mathcal{U}=\left\{U_{1}, \ldots, U_{k}\right\} \subset \mathbb{M}_{\text {sym }}^{3 \times 3}$, where the matrices $U_{i}$ are positivedefinite and satisfy $\operatorname{det} U_{i}=\delta>0$. Assume that there exists $\mu>0$ and $v \in S^{2}$ such that $U_{i} v=\mu v$ for $i=1, \ldots, k$. Let $K=\mathrm{SO}(3) U_{1} \cup \cdots \cup \operatorname{SO}(3) U_{k}$. Then $K^{(2)}=K^{\mathrm{Ic}}=K^{\mathrm{rc}}=K^{\mathrm{qc}}=K^{\mathrm{pc}}$. Further, if

$$
\left\{U_{1}, \ldots, U_{n}\right\}=\left\{U_{i} \in \mathcal{U}:\left|U_{i} \tilde{e}\right|^{2}>\max _{j \neq i}\left|U_{j} \tilde{e}\right|^{2} \quad \text { for some } \tilde{e} \in S^{2}\right\},
$$

then there exists a set $\mathcal{E}_{n}=\left\{e_{1}, \ldots, e_{n}\right\} \subset S^{2}$ such that any of these hulls is given by

$$
\left\{F \in \mathbb{M}^{3 \times 3}: \operatorname{det} F=\delta, F^{\mathrm{T}} F v=\mu^{2} v,\left|F e_{i}\right|^{2} \leqslant \max _{j=1, \ldots, n}\left|U_{j} e_{i}\right|^{2}, i=1, \ldots, n\right\} .
$$

Applications in the recently developed theory of thin films $[7,15]$ motivate to consider the following set $K$. 
TheOREM 1.3. Assume that $U_{i} \in \mathbb{M}_{\mathrm{sym}}^{3 \times 3}, i=1, \ldots, k$, are positive-definite with $\operatorname{adj}_{33} U_{i}^{2}=\delta^{2}>0$ and that $\left\{e_{1}, e_{2}, e_{3}\right\}$ is the standard orthonormal basis in $\mathbb{R}^{3}$. Let $K=\mathrm{SO}(3) \hat{U}_{1} \cup \cdots \cup \mathrm{SO}(3) \hat{U}_{k}$, where

$$
\mathrm{SO}(3) \hat{U}_{i}=\left\{Q \hat{U}_{i}=\left(Q U_{i} e_{1}, Q U_{i} e_{2}\right): Q \in \mathrm{SO}(3)\right\} \subset \mathbb{M}^{3 \times 2} .
$$

Then $K^{(3)}=K^{\mathrm{lc}}=K^{\mathrm{rc}}=K^{\mathrm{qc}}=K^{\mathrm{pc}}$ and any of these hulls is given by

$$
\left\{F \in \mathbb{M}^{3 \times 2}: \operatorname{det}\left(F^{\mathrm{T}} F\right) \leqslant \delta^{2},|F e|^{2} \leqslant \max _{i=1, \ldots, k}\left|\hat{U}_{i} e\right|^{2} \forall e \in S^{1}\right\} .
$$

We use this characterization to propose 'approximate relaxed energies', which may be useful for numerical computations. Minimizing sequences and minimizers of $I$ develop complex oscillatory patterns and this makes numerical computations challenging. Computing with the relaxed energy $I^{\#}$ (which is obtained from $I$ by replacing $W$ with its quasiconvex envelope) is attractive. Many of the numerical difficulties do not arise, the infima coincide, the minimizing sequences of $I$ converge to the minimizers of $I^{\#}$, and recently Ball et al. [5] have shown that under suitable growth hypotheses even the stresses associated with the minimizing sequences of $I$ converge to those associated with the minimizers of $I^{\#}$. Unfortunately, the quasiconvex envelope of $W$ is unknown. However, the practical interest lies in the behaviour of the quasiconvex envelope near the set $K^{\mathrm{qc}}$. We use the characterization of this set to propose functions $\bar{W}$ which are quasiconvex, vanish on $K^{\mathrm{qc}}$ and grow quadratically away from $K^{\mathrm{qc}}$. In [6] we adapt the construction to fit measured elastic moduli for various materials.

We illustrate our results with two examples.

(i) The two-well problem, which corresponds to an orthorhombic to monoclinic transformation and also arises under suitable assumptions in cubic to tetragonal or cubic to orthorhombic transformations, is described in examples 3.4 and 4.4 (example 3.4 recovers the results of Ball and James [4]).

(ii) The four-well problem, which corresponds to a tetragonal to monoclinic transformation and also arises under suitable assumptions in some cubic to monoclinic transformations, is described in examples 3.7, 4.57 .3 and 8.3.

Müller and Šverák [16,17] recently showed (based on Gromov's idea of convex integration) that there exist even Lipschitz continuous minimizers of $I$ if $F$ belongs to the interior of the rank-one convex hull of $K$ and if $K$ admits an 'in-approximation' (see $\S 6$ below for the precise statement); Dacarogna and Marcellini $[10,11]$ have obtained similar existence results using Baire's theorem. We show that the sets $K^{\mathrm{qc}}$ in theorems 1.1 and 1.3 , but not in theorem 1.2 , admit such in-approximations.

The basic ideas behind the main results are simple, though the details are rather laborious. Two identifications play a crucial role. First, $K$, and consequently the quasiconvex hull $K^{\mathrm{qc}}$, is invariant under (multiplication from the left by elements of) $\mathrm{SO}(2), \mathrm{O}(2)$ and $\mathrm{SO}(3)$. So we can look at the image $K_{\mathrm{c}}^{\mathrm{qc}}$ of $K^{\mathrm{qc}}$ in the space of $2 \times 2$ positive-semidefinite symmetric matrices under the map $F \mapsto F^{\mathrm{T}} F$. In other words, we identify the set

$$
K_{\mathrm{c}}^{\mathrm{qc}}=\left\{C \in \mathbb{M}_{\mathrm{sym}}^{2 \times 2}: \operatorname{det} C \geqslant 0, \sqrt{ } C \in K^{\mathrm{qc}}\right\}
$$




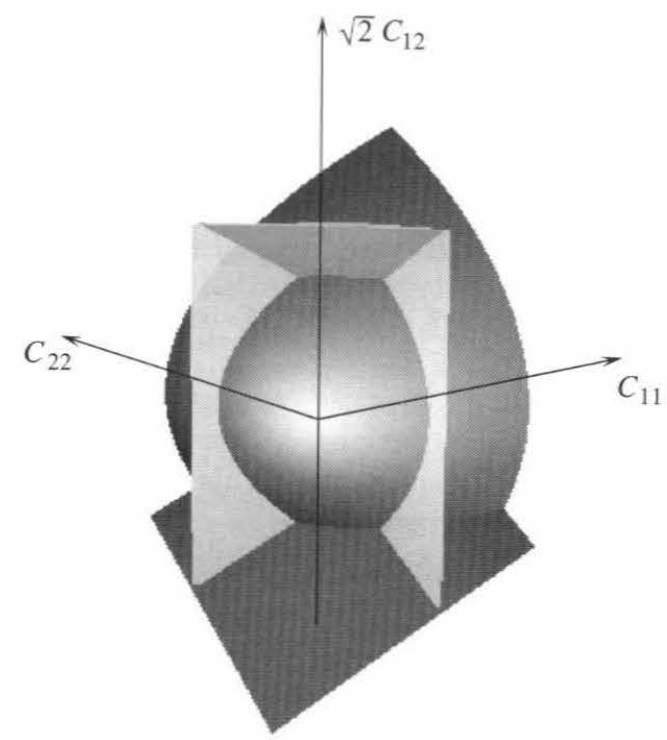

Figure 1. The quasiconvex hull $K_{\mathrm{c}}^{\mathrm{qc}}$ for the four-well problem described in example 3.7 with $\mathrm{SO}(2)$-invariant wells.

with $K^{\mathrm{qc}}$. Second, we identify the space $\mathbb{M}_{\text {sym }}^{2 \times 2}$ of symmetric $2 \times 2$ matrices with $\mathbb{R}^{3}$ using components $\left\{C_{11}, C_{22}, \sqrt{2} C_{12}\right\}$. We use the $\sqrt{ } 2$ in the third component to preserve inner products. Positive-semidefinite symmetric matrices correspond to the (affine) half cone

$$
\left\{C: C_{11} C_{22}-C_{12}^{2} \geqslant 0, C_{11} \geqslant 0, C_{22} \geqslant 0\right\} .
$$

We now give a brief non-technical discussion of our results. Under the assumptions in theorem 1.1(i), it follows from the minors relation or the weak continuity of the minors that, for any $F \in K^{\mathrm{qc}}$ and $e \in S^{1}, \operatorname{det} F=\delta$ and $|F e|^{2} \leqslant \max _{i=1, \ldots, k}\left|U_{i} e\right|^{2}$. Therefore, $K_{\mathrm{c}}^{\mathrm{qc}} \subset \mathcal{A}$, where

$$
\mathcal{A}=\left\{C \in \mathbb{M}_{\mathrm{sym}}^{2 \times 2}: \operatorname{det} C=\delta^{2},\langle e, C e\rangle \leqslant \max _{i=1, \ldots, k}\left|U_{i} e\right|^{2} \forall e \in S^{1}\right\} .
$$

We now show the converse, $\mathcal{A} \subset K_{\mathrm{c}}^{\mathrm{qc}}$. In order to do so, let us look at this set $\mathcal{A}$ in some detail. Clearly, the set of all positive-definite symmetric matrices with $\operatorname{det} C=\delta^{2}$ describes a manifold (hyperboloid), while $\langle e, C e\rangle=\alpha$ defines a plane in $\mathbb{R}^{3}$. Thus $\mathcal{A}$ is a subset of this manifold restricted by suitable planes (see figure 1 ). Let us elaborate. Figure 2 shows schematically the surface of the hyperboloid. For any direction $e \in S^{1}$ and $\alpha \in \mathbb{R}$, the intersection of the hyperboloid with the plane $\langle e, C e\rangle=\alpha$ is a (quadratic) curve $\Gamma(e, \alpha)$, which divides the hyperboloid into two parts (see the lower left of figure 2). Start with $\alpha \geqslant \max _{i=1, \ldots, k}\left|U_{i} e\right|^{2}$ and move the curve (by changing $\alpha$ ) until it first touches any of the matrices $U_{i}^{2}$. The set $\mathcal{A}$ is the set that is enclosed by similar curves for all $e \in S^{1}$. It turns out that if there are $k$ matrices, only $k$ curves are needed to define the boundary of $\mathcal{A}$ (of course, this requires a hypothesis that prevents one of the matrices $U_{i}^{2}$ to lie within the set $\mathcal{A}$ defined using the others; otherwise there may be less than $k$ curves). These $k$ curves have the property that they pass through two points $U_{i}^{2}$ and $U_{j}^{2}$. Further, 




Figure 2. The details of the quasiconvex hull $K_{\mathrm{c}}^{\mathrm{qc}}$ for the four-well problem described in example 3.7 with $\mathrm{SO}(2)$-invariant wells.

they are rank-one directions in the following sense. We can find $a, n \in \mathbb{R}^{2}$ such that any $C$ on this curve can be expressed as

$$
C=\left(U_{i}+t a \otimes n\right)^{\mathrm{T}}\left(U_{i}+t a \otimes n\right), \quad t \in \mathbb{R} .
$$

Therefore, we can obtain any point $C$ on the segment of this curve between $U_{i}^{2}$ and $U_{j}^{2}$ by rank-one lamination and thus $\partial \mathcal{A} \subset K_{\mathrm{c}}^{\mathrm{qc}}$. Now pick any point $D$ in the interior of $\mathcal{A}$. There is a rank-one curve passing through $D$ which always lies on the hyperboloid and extends off to infinity in both directions. Therefore, it must intersect $\partial \mathcal{A}$ at two points, and we can obtain $D$ through the lamination of these points. We thus conclude that $\mathcal{A} \subset K_{\mathrm{c}}^{\mathrm{qc}}$.

The result and proof of theorem 1.2 are similar; we use the minors relations to prove one inclusion and lift the constructions above to three dimensions to prove the other.

Let us now turn to part (ii) of theorem 1.1, where $K$ consists of $k$ copies of $\mathrm{O}(2)$. The fundamental difference between this and the former case can be seen in the special case $k=1$. While $K^{\mathrm{qc}}$ for $K=\mathrm{SO}(2)$ is trivial, i.e. $K^{\mathrm{qc}}=\mathrm{SO}(2)$ or $K_{\mathrm{c}}^{\mathrm{qc}}=\{I\}, K^{\mathrm{qc}}$ for $K=\mathrm{O}(2)$ is the set of matrices with singular values in $[0,1]$,

$$
K^{\mathrm{qc}}=\left\{F: 0 \leqslant \lambda_{1}\left(F^{\mathrm{T}} F\right) \leqslant \lambda_{2}\left(F^{\mathrm{T}} F\right) \leqslant 1\right\}
$$

where $\lambda_{1}, \lambda_{2}$ are the eigenvalues so that

$$
K_{\mathrm{c}}^{\mathrm{qc}}=\left\{C: C_{11} C_{22}-C_{12}^{2} \geqslant 0,\left(C_{11}-1\right)\left(C_{22}-1\right)-C_{12}^{2} \geqslant 0\right\} .
$$

The set $K_{\mathrm{c}}^{\mathrm{qc}}$ is shown in figure 3 and is obtained as the intersection of two back-toback cones given by the two inequalities above, one with apex $C=0$ and another 




Figure 3. The quasiconvex hull $K_{\mathrm{c}}^{\mathrm{qc}}$ for $K=\mathrm{O}(2)$.



Figure 4. The quasiconvex hull $K_{\mathrm{c}}^{\mathrm{qc}}$ for the four-well problem described in example 4.5 with $\mathrm{O}(2)$-invariant wells.

with apex $C=I$. This is due to the fact that $\mathrm{O}(2)$ consists of two copies of $\mathrm{SO}(2)$ which have remarkably many rank-one connections: any $Q \in \mathrm{O}(2) \backslash \mathrm{SO}(2)$ is rankone connected to the identity matrix $I$. For $k>1$, the set $K_{\mathrm{c}}^{\mathrm{qc}}$ is obtained by combining figures 1 and 3 , i.e. by composing the matrices in $\mathcal{A}$ with short maps. This set is shown in figure 4 , and the boundary consists of $\mathcal{A}$, the cone $\operatorname{det} C=0$ with apex at $C=0$, the planes $\langle e, C e\rangle=\max _{i=1, \ldots, k}\left|U_{i} e\right|^{2}$ and portions of cones with apexes at $U_{1}^{2}, \ldots, U_{k}^{2}$. The proof is very similar to that of theorem 1.1 (i); we use the minors relation to find bounds on $K_{\mathrm{c}}^{\mathrm{qc}}$ and use lamination to show that these bounds are indeed optimal. Finally, note that, unlike part (i), where it is sufficient to use only a finite number of directions $e$ to define the set $K^{\mathrm{qc}}$, in part (ii) we 
need all directions $e \in S^{1}$. A finite subset corresponds to the planar parts of the boundary of $K_{\mathrm{c}}^{\mathrm{qc}}$, while the rest define the cones with apexes at $U_{i}^{2}$.

The set $K_{\mathrm{c}}^{\mathrm{qc}}$, when $K$ consists of $k$ copies of $\mathrm{SO}(3) \hat{U}_{i}$, is described in theorem 1.3, and is identical to the case when $K$ consists of $k$ copies of $\mathrm{O}(2)$.

The paper is organized as follows. Section 2 collects preliminaries and basic lemmas, which are used in the subsequent sections. We suggest that a reader omit it on first reading, coming back to it as and when necessary. Theorems 1.1-1.3 will be proven and illustrated with examples in $\S \S 3-5$. Section 6 discusses existence of minimizers, while we present in $\S 7$ uniqueness and non-uniqueness results for microstructures associated with minimizing sequences for the variational problem (1.1). In $\S 8$ we finally construct approximate relaxed energies.

\section{Preliminaries}

The generalized convex hulls we are concerned with in this paper are defined as sublevel sets of functions with the corresponding convexity properties. Recall that a function $f: \mathbb{M}^{2 \times 2} \rightarrow(-\infty, \infty]$ is said to be convex if

$$
f(\lambda A+(1-\lambda) B) \leqslant \lambda f(A)+(1-\lambda) f(B) \quad \forall A, B \in \mathbb{M}^{2 \times 2}, \quad \lambda \in(0,1),
$$

and it is said to be rank-one convex if (2.1) holds for all $A, B \in \mathbb{M}^{2 \times 2}$ with $\operatorname{rank}(A-B)=1$. Rank-one convexity is a necessary condition for quasiconvexity, the fundamental notion of convexity in the calculus of variations. A function $f$ is quasiconvex if

$$
\int_{B(0,1)} f(F+D \varphi) \mathrm{d} x \geqslant \int_{B(0,1)} f(F) \mathrm{d} x \quad \forall \varphi \in W_{0}^{1, \infty}\left(B(0,1) ; \mathbb{R}^{2}\right)
$$

(whenever the integral on the left-hand side exists). A sufficient condition for quasiconvexity is polyconvexity, i.e. there exists a convex function $g: \mathbb{R}^{5} \rightarrow \mathbb{R}$ such that $f(F)=g(F, \operatorname{det} F)$. We now define for a given compact set $K \subset \mathbb{M}^{2 \times 2}$ its rank-one convex hull $K^{\mathrm{rc}}$ by

$$
K^{\mathrm{rc}}=\left\{F \in \mathbb{M}^{2 \times 2}: f(F) \leqslant \sup _{K} f \text { for all } f: \mathbb{M}^{2 \times 2} \rightarrow \mathbb{R} \text { rank-one convex }\right\} .
$$

The quasiconvex hull $K^{\mathrm{qc}}$, the polyconvex hull $K^{\mathrm{pc}}$ and the convex hull $K^{\mathrm{c}}$ are defined analogously. Finally, we define the lamination convex hull $K^{\mathrm{lc}}$ in the following way (see [16]). Let $K^{(0)}=K$ and define

$$
K^{(i+1)}=\left\{\lambda A+(1-\lambda) B: A, B \in K^{(i)}, \operatorname{rank}(A-B)=1, \lambda \in(0,1)\right\} \cup K^{(i)} .
$$

Then

$$
K^{\mathrm{lc}}=\bigcup_{i=0}^{\infty} K^{(i)}
$$

It follows that

$$
K^{\mathrm{lc}} \subset K^{\mathrm{rc}} \subset K^{\mathrm{qc}} \subset K^{\mathrm{pc}} \subset K^{\mathrm{c}}
$$

(see $[9,19])$. 
We now introduce some notation that we frequently use. Given distinct matrices $U_{1}, \ldots, U_{k} \in \mathbb{M}_{\text {sym }}^{2 \times 2}$, we let

$$
\mathcal{U}=\left\{U_{1}, \ldots, U_{k}\right\} .
$$

We note that if $\operatorname{det} U_{i}=\delta>0$ for $i=1, \ldots, k$, then, according to the polar decomposition theorem,

$$
U_{j} \notin \bigcup_{i \neq j} \mathrm{SO}(2) U_{i}
$$

for $j=1, \ldots, k$, so that the $\mathrm{SO}(2)$ wells are disjoint. We often use

$$
m_{\mathcal{U}}(e)=\max \left\{|U e|^{2}: U \in \mathcal{U}\right\} .
$$

We denote by $e^{\perp}$ the unique unit vector orthogonal to $e \in S^{1}$ with $\operatorname{det}\left(e, e^{\perp}\right)=1$.

We collect in the next two lemmas well-known facts (see [12]), which will be useful throughout the paper.

Lemma 2.1. Assume that $C_{1}, C_{2} \in \mathbb{M}_{\text {sym }}^{2 \times 2}$ are positive-semidefinite, $C_{1}=F_{1}^{\mathrm{T}} F_{1}$, $C_{2}=F_{2}^{\mathrm{T}} F_{2}$. Let $e \in S^{1}$. Then the following four statements are equivalent.

(i) There exist $Q \in \mathrm{SO}(2)$ and $a \in \mathbb{R}^{2}$ such that $Q F_{1}-F_{2}=a \otimes e^{\perp}$.

(ii) We have $\left|F_{1} e\right|^{2}=\left|F_{2} e\right|^{2}$.

(iii) There exists $v \in \mathbb{R}^{2}$ such that $C_{1}=C_{2}+v \otimes e^{\perp}+e^{\perp} \otimes v$.

(iv) $\operatorname{det}\left(C_{1}-C_{2}\right) \leqslant 0$.

Moreover, the vector a in statement (i) and the vector $v$ in statement (iii) are related by $v=F_{2}^{\mathrm{T}} a+\frac{1}{2}|a|^{2} e^{\perp}$. Finally, if $\operatorname{det} F_{1}=\operatorname{det} F_{2}$, then $a=\alpha F_{2} e$ with $\alpha \in \mathbb{R}$.

Proof. (i) $\Rightarrow$ (ii). Assume that $Q F_{1}=F_{2}+a \otimes e^{\perp}$. Then

$$
\begin{aligned}
C_{1} & =\left(F_{2}^{\mathrm{T}}+e^{\perp} \otimes a\right)\left(F_{2}+a \otimes e^{\perp}\right) \\
& =C_{2}+F_{2}^{\mathrm{T}} a \otimes e^{\perp}+e^{\perp} \otimes F_{2}^{\mathrm{T}} a+|a|^{2} e^{\perp} \otimes e^{\perp}
\end{aligned}
$$

and (ii) follows immediately.

(ii) $\Rightarrow$ (iii). Let $\bar{C}=C_{1}-C_{2}$. Assume first that $\operatorname{rank}(\bar{C})=1$. Since $\bar{C}$ is symmetric, there exists a $v \in \mathbb{R}^{2}$ such that $\bar{C}=v \otimes v$. By assumption, $\langle e, \bar{C} e\rangle=\langle v, e\rangle^{2}=0$ and thus we obtain (iii) with $v$ parallel to $e^{\perp}$.

Consider now the case $\operatorname{rank}(\bar{C})=2$. Since the eigenvalues $\lambda_{i}$ of $\bar{C}$ satisfy

$$
\lambda_{1}(\bar{C})=\min _{v \in S^{1}}\langle v, \bar{C} v\rangle<0<\max _{v \in S^{1}}\langle v, \bar{C} v\rangle=\lambda_{2}(\bar{C}),
$$

there exist $\alpha_{1}, \alpha_{2} \in \mathbb{R} \backslash\{0\}$ and orthonormal vectors $v_{1}, v_{2} \in \mathbb{R}^{2}$ such that

$$
\begin{aligned}
\bar{C} & =\alpha_{2}^{2} v_{2} \otimes v_{2}-\alpha_{1}^{2} v_{1} \otimes v_{1} \\
& =\frac{1}{2}\left\{\left(\alpha_{2} v_{2}-\alpha_{1} v_{1}\right) \otimes\left(\alpha_{2} v_{2}+\alpha_{1} v_{1}\right)+\left(\alpha_{2} v_{2}+\alpha_{1} v_{1}\right) \otimes\left(\alpha_{2} v_{2}-\alpha_{1} v_{1}\right)\right\} .
\end{aligned}
$$

Clearly, $\left\langle e, v_{1}\right\rangle \neq 0$ and $\left\langle e, v_{2}\right\rangle \neq 0$ (indeed, if $\left\langle e, v_{1}\right\rangle=0$, then $v_{2}=\gamma e$, with $\gamma \in\{ \pm 1\}$ and $e \bar{C} e=\alpha_{2}^{2} \neq 0$; a contradiction). This implies

$$
\left\langle e, v_{2}\right\rangle^{2}=\left(\alpha_{1}^{2} / \alpha_{2}^{2}\right)\left\langle e, v_{1}\right\rangle^{2} ;
$$


since $\left\{v_{1}, v_{2}\right\}$ is an orthonormal basis of $\mathbb{R}^{2}$, we infer

$$
e=\left\langle e, v_{1}\right\rangle v_{1}+\left\langle e, v_{2}\right\rangle v_{2}=\frac{\left\langle e, v_{1}\right\rangle}{\alpha_{2}}\left(\alpha_{2} v_{1} \pm \alpha_{1} v_{2}\right)
$$

and

$$
e^{\perp}=\frac{\left\langle e, v_{1}\right\rangle}{\alpha_{2}}\left(\mp \alpha_{1} v_{1}+\alpha_{2} v_{2}\right) .
$$

This proves (iii) with $v=\alpha_{2} v_{2} \pm \alpha_{1} v_{1}$.

(iii) $\Rightarrow$ (ii). This is obvious.

(ii) $\Rightarrow$ (i). By assumption, $\left|F_{1} e\right|^{2}=\left|F_{2} e\right|^{2}$ and we may choose $Q \in \mathrm{SO}(2)$ such that $Q F_{1} e=F_{2} e$ or $\left(Q F_{1}-F_{2}\right) e=0$. Thus $Q F_{1}-F_{2}=a \otimes n$ is a matrix of rank one and from $\left(Q F_{1}-F_{2}\right) e=\langle n, e\rangle a=0$ we deduce that we may choose $n=e^{\perp}$.

(ii) $\Leftrightarrow$ (iv). This is also obvious from above (the characterization of $\bar{C}$ ).

The relation between $a$ in statement (i) and $v$ in statement (iii) follows by direct calculation. Finally, if $\operatorname{det} F_{1}=\operatorname{det} F_{2}$, then (i) implies

$$
\operatorname{det} F_{1}=\left(\operatorname{det} F_{2}\right)\left(1+\left\langle F_{2}^{-1} a, e^{\perp}\right\rangle\right)
$$

and thus $a$ must be parallel to $F_{2} e$.

Lemma 2.2. If $C_{1}, C_{2} \in \mathbb{M}_{\text {sym }}^{2 \times 2}, C_{1}=F_{1}^{\mathrm{T}} F_{1}$ and $C_{2}=F_{2}^{\mathrm{T}} F_{2}$ are positive-definite with $\operatorname{det} C_{1}=\operatorname{det} C_{2}$, then there exist rotations $Q_{i} \in \mathrm{SO}(2)$ and vectors $a_{i}, n_{i} \in \mathbb{R}^{2}$, $i=1,2$, such that $n_{1}$ and $n_{2}$ are not parallel and $Q_{i} F_{1}-F_{2}=a_{i} \otimes n_{i}$. Moreover, $\left|\left(\lambda Q_{1} F_{1}+(1-\lambda) F_{2}\right) n_{2}^{\frac{1}{2}}\right|^{2}<\left|F_{1} n_{2}^{\frac{1}{2}}\right|^{2}$ for $\lambda \in(0,1)$.

Proof. Denote by $\lambda_{1} \leqslant \lambda_{2}$ and $\mu_{1} \leqslant \mu_{2}$ the eigenvalues of $C_{1}$ and $C_{2}$. Since, by hypothesis, $\operatorname{det} C_{1}=\operatorname{det} C_{2}$, or $\lambda_{1} \lambda_{2}=\mu_{1} \mu_{2}$, we may assume that $\lambda_{1} \leqslant \mu_{1}$ and $\mu_{2} \leqslant \lambda_{2}$, or

$$
\min _{w \in S^{1}}\left\langle w, C_{1} w\right\rangle \leqslant \min _{w \in S^{1}}\left\langle w, C_{2} w\right\rangle \text { and } \max _{w \in S^{1}}\left\langle w, C_{1} w\right\rangle \geqslant \max _{w \in S^{1}}\left\langle w, C_{2} w\right\rangle .
$$

Therefore, we can deduce from the continuity of the mappings $w \mapsto\left\langle w, C_{1} w\right\rangle$ and $w \mapsto\left\langle w, C_{2} w\right\rangle$ that there exists a $w_{1}$ such that $\left\langle w_{1}, C_{1} w_{1}\right\rangle=\left\langle w_{1}, C_{2} w_{1}\right\rangle$. By lemma 2.1 , there exists $w_{2} \in \mathbb{R}^{2}$ such that $C_{1}=C_{2}+w_{1}^{\perp} \otimes w_{2}+w_{2} \otimes w_{1}^{\perp}$. The existence of the rank-one connections now follows with $n_{1}=w_{1}^{\perp}$ and $n_{2}=w_{2} /\left|w_{2}\right|$ from the equivalence (i) $\Leftrightarrow$ (iii) in lemma 2.1. The vectors $w_{1}^{\perp}$ and $w_{2}$, and consequently $n_{1}$ and $n_{2}$, are not parallel since

$$
\operatorname{det} C_{1}=\operatorname{det}\left(C_{2}+\gamma w_{1}^{\perp} \otimes w_{1}^{\perp}\right)=\left(\operatorname{det} C_{2}\right)\left(1+\gamma\left\langle w_{1}^{\perp}, C_{2}^{-1} w_{1}^{\perp}\right\rangle\right) \neq \operatorname{det} C_{2}
$$

in view of $\left\langle w, C_{2}^{-1} w\right\rangle \geqslant \mu_{2}^{-1}>0$. The existence of the rank-one connections follows now with $n_{1}=w_{1}^{\perp}$ and $n_{2}=w_{2}$ from the equivalence (i) $\Leftrightarrow$ (iii) in lemma 2.1.

To prove the inequality, note that

$$
\left|F_{1} n_{2}^{\perp}\right|^{2}=\left|Q_{1} F_{1} n_{2}^{\perp}\right|^{2}=\left|F_{2} n_{2}^{\perp}\right|^{2}+2\left\langle n_{1}, n_{2}^{\perp}\right\rangle\left\langle F_{2} n_{2}^{\perp}, a_{1}\right\rangle+\left|a_{1}\right|^{2}\left(\left\langle n_{1}, n_{2}^{\perp}\right\rangle\right)^{2} .
$$

By lemma 2.1, $\left|F_{1} n_{2}^{\frac{1}{2}}\right|^{2}=\left|F_{2} n_{2}^{\frac{1}{2}}\right|^{2}$, so that

$$
2\left\langle n_{1}, n_{2}^{\perp}\right\rangle\left\langle F_{2} n_{2}^{\perp}, a_{1}\right\rangle+\left|a_{1}\right|^{2}\left(\left\langle n_{1}, n_{2}^{\perp}\right\rangle\right)^{2}=0 .
$$


Note that $\left\langle n_{1}, n_{2}^{\perp}\right\rangle \neq 0$, since $n_{1}$ and $n_{2}$ are not parallel. Therefore, $\alpha=-\beta<0$, where $\alpha=2\left\langle n_{1}, n_{2}^{\frac{1}{2}}\right\rangle\left\langle F_{2} n_{2}^{\perp}, a_{1}\right\rangle$ and $\beta=\left|a_{1}\right|^{2}\left\langle n_{1}, n_{2}^{\frac{1}{2}}\right\rangle$. Finally, a calculation shows that

$$
\left|\left(\lambda Q_{1} F_{1}+(1-\lambda) F_{2}\right) n_{2}^{\frac{1}{2}}\right|^{2}<\left|F_{1} n_{2}^{\perp}\right|^{2} \quad \Longleftrightarrow \quad \lambda \alpha+\lambda^{2} \beta=\lambda(1-\lambda) \alpha<0,
$$

and we obtain the assertion of the lemma.

Our characterization of the image of the generalized convex hulls in the threedimensional affine space of symmetric matrices uses the following property of the intersection of the surface $\left\{C: C\right.$ positive-definite, $\left.\operatorname{det} C=\delta^{2}\right\}$, with the twodimensional hyperplanes $\left\langle\left(C_{11}, C_{22}, \sqrt{2} C_{12}\right),\left(e_{1}^{2}, e_{2}^{2}, \sqrt{2} e_{1} e_{2}\right)\right\rangle=\gamma^{2}$.

Lemma 2.3. Assume that $e \in S^{1}$ and $\gamma, \delta \in \mathbb{R}, \delta>0$. Then the set

$$
\Gamma\left(e ; \gamma^{2}\right)=\left\{F^{\mathrm{T}} F:(\operatorname{det} F)^{2}=\delta^{2},|F e|^{2}=\gamma^{2}\right\} \subset \mathbb{R}^{3}
$$

is either empty or a smooth one-dimensional manifold which can be parametrized by $t \mapsto F_{t}^{\mathrm{T}} F_{t}$ with $F_{t}=F\left(I+t e \otimes e^{\perp}\right)$ for any $F \in \Gamma(e ; \gamma)$.

Proof. Let $E=\left\{F^{\mathrm{T}} F: \operatorname{det} F \neq 0\right\} \subset \mathbb{R}^{3}$ and define $\Phi: E \rightarrow \mathbb{R}^{2}$ by

$$
\Phi(X)=\left(\begin{array}{c}
X_{11} X_{22}-X_{12}^{2}-\delta^{2} \\
e_{1}^{2} X_{11}+e_{2}^{2} X_{22}+2 e_{1} e_{2} X_{12}-\gamma^{2}
\end{array}\right) .
$$

It is easy to see that rank $D \Phi=2$ on $E$ and thus $\Phi^{-1}(0)$ is a smooth one-dimensional manifold contained in $E$. Assume that $\Gamma(e ; \gamma) \neq \emptyset$ and let $F_{0}^{\mathrm{T}} F_{0} \in \Gamma(e ; \gamma)$. Any $F^{\mathrm{T}} F \in \Gamma(e ; \gamma)$ satisfies $|F e|^{2}=\left|F_{0} e\right|^{2}$ and thus there exists by lemma 2.1 an $\alpha \in \mathbb{R}$ such that $F=F_{0}\left(I+\alpha e \otimes e^{\perp}\right)$. This proves the assertion of the lemma.

The next lemmas will be important ingredients for the characterization of the boundaries of the generalized convex hulls. Recall that $m_{\mathcal{U}}$ has been defined in (2.3).

Lemma 2.4. Suppose $F \in \mathbb{M}^{2 \times 2}$ satisfies the following conditions.

(i) $|F e|^{2} \leqslant m_{\mathcal{U}}(e)$ for all $e \in S^{1}$.

(ii) There exist $\tilde{e} \in S^{1}$ and $i \in\{1, \ldots, k\}$ such that $|F \tilde{e}|^{2}=\left|U_{i} \tilde{e}\right|^{2}>m_{\mathcal{U} \backslash\left\{U_{i}\right\}}(\tilde{e})$.

Then there exists an $\alpha \in \mathbb{R}$ such that $F^{\mathrm{T}} F=U_{i}^{2}-\alpha^{2} \tilde{e}^{\perp} \otimes \tilde{e}^{\perp}$. Moreover, if $\operatorname{det} F=\delta$, then $F=Q U_{i}$ for some $Q \in \mathrm{SO}(2)$.

Proof. In view of lemma 2.1, there exists a $v \in \mathbb{R}^{2}$ such that

$$
F^{\mathrm{T}} F=U_{i}^{2}+v \otimes \tilde{e}^{\perp}+\tilde{e}^{\perp} \otimes v .
$$

Let $e_{\theta}=\left(1+\theta^{2}\right)^{-1 / 2}\left(\tilde{e}+\theta \tilde{e}^{\perp}\right)$. By assumption, we may choose $\varepsilon>0$ small enough such that

Thus

$$
m_{\mathcal{U} \backslash\left\{U_{i}\right\}}\left(e_{\theta}\right)<\left|F e_{\theta}\right|^{2} \leqslant\left|U_{i} e_{\theta}\right|^{2} \text { for }|\theta|<\varepsilon .
$$

$$
\left\langle e_{\theta},\left(v \otimes \tilde{e}^{\perp}+\tilde{e}^{\perp} \otimes v\right) e_{\theta}\right\rangle=\left\langle v, e_{\theta}\right\rangle\left\langle\tilde{e}^{\perp}, e_{\theta}\right\rangle=\frac{\theta}{\sqrt{1+\theta^{2}}}\left\langle v, e_{\theta}\right\rangle \leqslant 0 .
$$


We conclude that

$$
\sqrt{1+\theta^{2}} \theta\left\langle v, e_{\theta}\right\rangle=\theta\langle\tilde{e}, v\rangle+\theta^{2}\left\langle\tilde{e}^{\perp}, v\right\rangle \leqslant 0
$$

and this implies $v=\gamma \tilde{e}^{\perp}$ with $\gamma \leqslant 0$. Thus $F^{\mathrm{T}} F=U_{i}^{2}-\alpha^{2} \tilde{e}^{\perp} \otimes \tilde{e}^{\perp}$. If $\operatorname{det} F=\delta$, then $\delta^{2}=\delta^{2}\left(1-\alpha^{2}\left|U_{i}^{-\mathrm{T}} \tilde{e}^{\perp}\right|^{2}\right)$, and therefore $\alpha=0$. This implies the assertion of the lemma.

LEMma 2.5. Suppose there exists $e \in S^{1}$ and $2 \leqslant n \leqslant k$ such that

$$
\left|U_{1} e\right|^{2}=\cdots=\left|U_{n} e\right|^{2}=m_{\mathcal{U}}(e)>\max \left\{\left|U_{i} e\right|^{2}: i=n+1, \ldots, k\right\} .
$$

Set $e_{\theta}=\left(1+\theta^{2}\right)^{-1 / 2}\left(e+\theta e^{\perp}\right)$. Then there exist $p, q \in\{1, \ldots, n\}, p \neq q$ and $\theta_{0}>0$ such that the following three statements hold.

(i) $m_{\mathcal{U}}\left(e_{\theta}\right)=\left|U_{p} e_{\theta}\right|^{2}>m_{\mathcal{U} \backslash\left\{U_{p}\right\}}\left(e_{\theta}\right)$ for $-\theta_{0}<\theta<0$.

(ii) $m_{\mathcal{U}}\left(e_{\theta}\right)=\left|U_{q} e_{\theta}\right|^{2}>m_{\mathcal{U} \backslash\left\{U_{q}\right\}}\left(e_{\theta}\right)$ for $0<\theta<\theta_{0}$.

(iii) For all $i \in\{1, \ldots, n\}$, we have

$$
U_{i} \in\left(\mathrm{SO}(2) U_{p} \cup \mathrm{SO}(2) U_{q}\right)^{(1)} .
$$

Proof. It follows from the continuity of the mappings $e \mapsto\left|U_{i} e\right|^{2}$ that there exists a $\theta_{0}>0$ such that

$$
m_{\mathcal{U}}\left(e_{\theta}\right)=\max _{i=1, \ldots, n}\left|U_{i} e_{\theta}\right|^{2}>\max _{i=n+1, \ldots, k}\left|U_{i} e_{\theta}\right|^{2} \quad \text { for }|\theta|<\theta_{0} .
$$

By lemma 2.3 (with $F=U_{1}$ ), there exist $t_{i} \in \mathbb{R}, i=1, \ldots, n$, such that

$$
U_{i}^{2}=U_{1}^{2}+t_{i}\left(U_{1}^{2} e \otimes e^{\perp}+e^{\perp} \otimes U_{1}^{2} e\right)+t_{i}^{2}\left|U_{1}^{2} e\right|^{2} e^{\perp} \otimes e^{\perp} .
$$

Relabelling the matrices if necessary, we may assume that $t_{1}=0$ and $t_{i}>0$ for $i=2, \ldots, n$. Thus

$$
\left|U_{i} e_{\theta}\right|^{2}=\left|U_{1} e_{\theta}\right|^{2}+2 \frac{\theta t_{i}}{\sqrt{1+\theta^{2}}}\left|U_{1} e\right|^{2}+\frac{\theta^{2}}{1+\theta^{2}}\left(2 t_{i}\left\langle e^{\perp}, U_{1}^{2} e\right\rangle+t_{i}^{2}\left|U_{1}^{2} e\right|^{2}\right) .
$$

The conclusions (i) and (ii) follow with $t_{p}=t_{1}=0$ and $t_{q}=\max _{i=2, \ldots, n} t_{i}$ (where we choose $\theta_{0}$ sufficiently small).

To prove (iii), we assume that $p=1, i=2$ and $q=3$ (note that $t_{1}=0<t_{2}<t_{3}$ by construction of $p$ and $q)$. Let $C(t)=G(t)^{\mathrm{T}} G(t)$ with $G(t)=U_{1}\left(I+t e \otimes e^{\perp}\right)$. Then $C(0)=U_{1}^{\mathrm{T}} U_{1}, C\left(t_{1}\right)=U_{2}^{\mathrm{T}} U_{2}$ and $C\left(t_{2}\right)=U_{3}^{\mathrm{T}} U_{3}$. Let $V_{i}$ be the square root of $C_{i}$. By the polar decomposition theorem, there exist $Q_{i}, R_{i} \in \mathrm{SO}(2), i=1,2,3$, such that $Q_{i} V_{i}=G\left(t_{i}\right)$ and $Q_{i} V_{i}=R_{i} U_{i}$. Let $\lambda=t_{2} / t_{3}$. Then

$$
(1-\lambda) G\left(t_{1}\right)+\lambda G\left(t_{3}\right)=\frac{t_{3}-t_{2}}{t_{3}} U_{1}+\frac{t_{2}}{t_{3}} U_{1}\left(I+t_{2} e \otimes e^{\perp}\right)=G\left(t_{2}\right),
$$

and therefore $(1-\lambda) R_{2}^{\mathrm{T}} R_{1} U_{1}+\lambda R_{2}^{\mathrm{T}} R_{3} U_{3}=U_{2}$.

LEMma 2.6. Assume that $U_{i} \in \mathcal{U}$ and that there exists $\tilde{e} \in S^{1}$ such that

$$
\left|U_{i} \tilde{e}\right|^{2}=m_{\mathcal{U}}(\tilde{e})>m_{\mathcal{U} \backslash\left\{U_{i}\right\}}(\tilde{e}) .
$$

Then there exists $U_{j} \in \mathcal{U}, i \neq j$, and $e \in S^{1}$ such that $\left|U_{i} e\right|^{2}=\left|U_{j} e\right|^{2}=m_{\mathcal{U}}(e)$. 
Proof. Suppose the conclusion was wrong. Since $\left|U_{i} e\right|^{2}=\left|U_{i}(-e)\right|^{2}$, we may assume that all vectors $e \in S^{1}$ are given by $e=e(\varphi)=(\cos \varphi, \sin \varphi)$ with $\varphi \in[0, \pi)$. In particular, the map $\varphi \mapsto\left|U_{i} e(\varphi)\right|^{2}$ is a continuous periodic map on $[0, \pi]$. By assumption, either $\left|U_{i} e(\varphi)\right|^{2}>m_{\mathcal{U} \backslash\left\{U_{i}\right\}}(e(\varphi))$ or $\left|U_{i} e(\varphi)\right|^{2}<m_{\mathcal{U} \backslash\left\{U_{i}\right\}}(e(\varphi))$. Since the latter case is excluded by assumption, we conclude that the former holds. Choose any $U_{k} \in \mathcal{U}, U_{k} \neq U_{i}$. By lemma 2.2 , there exists $t \in \mathbb{R}, \bar{e} \in S^{1}$ and $Q \in \mathrm{SO}(2)$ such that $Q U_{i}-U_{k}=t U_{k} \bar{e} \otimes \bar{e}^{\perp}$ and $\left|U_{i} \bar{e}\right|^{2}=\left|U_{k} \bar{e}\right|^{2} \leqslant m_{\mathcal{U}}(\bar{e})$. This violates our hypothesis and we deduce that there exists at least one $e \in S^{1}$ and $U_{j} \in \mathcal{U}, U_{j} \neq U_{i}$ such that $\left|U_{i} e\right|^{2}=\left|U_{j} e\right|^{2}=m_{\mathcal{U}}(e)$.

Lemma 2.7. Assume that $U_{i}, U_{j} \in \mathcal{U}, i \neq j$, and that there exists $\varepsilon>0$ and $e_{1}=\left(\cos \varphi_{1}, \sin \varphi_{1}\right)$ with $\varphi_{1} \in[0, \pi)$ such that the following hold.

(i) $\left|U_{i} e_{1}\right|^{2}=\left|U_{j} e_{1}\right|^{2}=m_{\mathcal{U}}\left(e_{1}\right)$.

(ii) $m_{\mathcal{U}}(e(\varphi))=\left|U_{i} e(\varphi)\right|^{2}>m_{\mathcal{U} \backslash\left\{U_{i}\right\}}(e(\varphi))$ for $\varphi_{1}<\varphi<\varphi_{1}+\varepsilon$.

(iii) $m_{\mathcal{U}}(e(\varphi))=\left|U_{j} e(\varphi)\right|^{2}>m_{\mathcal{U} \backslash\left\{U_{j}\right\}}(e(\varphi))$ for $\varphi_{1}-\varepsilon<\varphi<\varphi_{1}$.

Then there exists $U_{m} \in \mathcal{U}, m \neq i$, and $e_{2} \in S^{1}$ not parallel to $e_{1}$ such that

$$
\left|U_{i} e_{2}\right|^{2}=\left|U_{m} e_{2}\right|^{2}=m_{\mathcal{U}}\left(e_{2}\right) .
$$

Proof. Define

$$
\tilde{\varphi}_{2}=\max \left\{\varphi \geqslant \varphi_{1}:\left|U_{i} e(\varphi)\right|^{2}=m_{\mathcal{U}}(e(\varphi)) \text { on }\left[\varphi_{1}, \varphi\right]\right\}
$$

and let $\varphi_{2}=\tilde{\varphi}_{2} \bmod \pi$. By (ii), $\tilde{\varphi}_{2}>\varphi_{1}$ and by (iii), we conclude that $\varphi_{2} \neq \varphi_{1}$. It follows that there exists $\delta>0$ such that

$$
m_{\mathcal{U}}(e(\varphi))>\left|U_{i}(e(\varphi))\right|^{2} \text { for } \varphi_{2}<\varphi<\varphi_{2}+\delta .
$$

The continuity of the mappings $\varphi \mapsto\left|U_{k}(e(\varphi))\right|^{2}$ implies the assertion of the lemma.

\section{The quasiconvex hull of $\mathrm{SO}(2) U_{1} \cup \cdots \cup \operatorname{SO}(2) U_{k}$}

In this section we prove theorems 1.1 (i) and 1.2 .

We first prove the following version of theorem 1.1, which uses an infinite number of inequalities to define $K^{\mathrm{qc}}$.

Proposition 3.1. Assume that $\left\{U_{1}, \ldots, U_{k}\right\} \subset \mathbb{M}_{\text {sym }}^{2 \times 2}$ with $U_{i}$ positive-definite and $\operatorname{det} U_{i}=\delta>0$. Let $K=\mathrm{SO}(2) U_{1} \cup \cdots \cup \mathrm{SO}(2) U_{k}$. Then

$$
K^{(2)}=K^{\mathrm{lc}}=K^{\mathrm{rc}}=K^{\mathrm{qc}}=K^{\mathrm{pc}}
$$

and any of these hulls is given by

$$
\left\{F: \operatorname{det} F=\delta,|F e|^{2} \leqslant \max _{j=1, \ldots, n}\left|U_{j} e\right|^{2} \forall e \in S^{1}\right\} .
$$


We split the proof of this proposition into a series of lemmas. Let

$$
\mathcal{A}=\left\{F \in \mathbb{M}^{2 \times 2}: \operatorname{det} F=\delta,|F e|^{2} \leqslant m_{\mathcal{U}}(e) \forall e \in S^{1}\right\} .
$$

We will show that $K^{\mathrm{pc}} \subset \mathcal{A} \subset K^{(2)}$. This proves the theorem, since, by (2.2), $K^{\mathrm{lc}} \subset K^{\mathrm{pc}}$.

Lemma 3.2. Suppose that the assumptions of theorem 1.1 hold and that $\mathcal{A}$ is defined by (3.1). Then $K^{\mathrm{pc}} \subset \mathcal{A}$.

Proof. We construct a polyconvex function $\Phi$, which vanishes on $\mathcal{A}$ and is positive elsewhere. Let $t_{+}=\max \{t, 0\}$ and define for $\nu \in S^{1}$ the function $g_{\nu}: \mathbb{M}^{2 \times 2} \rightarrow \mathbb{R}$ by

$$
g_{\nu}(X)=\left(|X \nu|^{2}-m_{\mathcal{U}}(\nu)\right)_{+} .
$$

Clearly, $g_{\nu}$ is convex since it is the composition of a convex non-decreasing function and a convex function. The supremum of convex functions is convex and therefore

$$
\Phi(X)=(\operatorname{det} X-\delta)^{2}+\sup _{\nu \in S^{1}} g_{\nu}(X)
$$

is the desired function.

The reverse inclusion $\mathcal{A} \subset K^{(2)}$ requires some preparation. Let

$$
\mathcal{B}=\left\{F: \operatorname{det} F=\delta,|F e|^{2} \leqslant m_{\mathcal{U}}(e) \forall e \in S^{1}, \exists \tilde{e}:|F \tilde{e}|^{2}=m_{\mathcal{U}}(\tilde{e})\right\} .
$$

As a first step we show in the next lemma that $\mathcal{B} \subset K^{(1)}$. Given $U_{i}, U_{j}$, according to lemma 2.2 there exists a $Q \in \mathrm{SO}(2)$ and $a, e \in \mathbb{R}^{2}$ such that $Q U_{j}-U_{i}=a \otimes e^{\perp}$. Let

$$
\Gamma_{i, j}\left(e ;\left|U_{i} e\right|^{2}\right)=\left\{\left(U_{i}+\lambda a \otimes e^{\perp}\right)^{\mathrm{T}}\left(U_{i}+\lambda a \otimes e^{\perp}\right): \lambda \in[0,1]\right\} \subset \mathbb{R}^{3}
$$

denote the arc connecting $U_{i}^{\mathrm{T}} U_{i}$ and $U_{j}^{\mathrm{T}} U_{j}$ on the curve $\Gamma\left(e ;\left|U_{i} e\right|^{2}\right)$.

Lemma 3.3. Assume that $k \geqslant 2$. Let $F \in \mathcal{B}$ and $C=F^{\mathrm{T}} F$.

(i) There exist $e \in S^{1}, U_{p}, U_{q} \in \mathcal{U}, p \neq q$, such that $\left|U_{p} e\right|^{2}=\left|U_{q} e\right|^{2}=m_{\mathcal{U}}(e)$ and $C \in \Gamma_{p, q}\left(e ; m_{\mathcal{U}}(e)\right)$. Moreover, we may choose $p$ and $q$ in such a way that there exist $\tilde{e}_{p}, \tilde{e}_{q} \in S^{1}$ such that $m_{\mathcal{U}}\left(\tilde{e}_{p}\right)=\left|U_{p} \tilde{e}_{p}\right|^{2}>m_{\mathcal{U} \backslash\left\{U_{p}\right\}}\left(\tilde{e}_{p}\right)$ and $m_{\mathcal{U}}\left(\tilde{e}_{q}\right)=\left|U_{q} \tilde{e}_{q}\right|^{2}>m_{\mathcal{U} \backslash\left\{U_{q}\right\}}\left(\tilde{e}_{q}\right)$.

(ii) We have $\mathcal{B} \subset K^{(1)}$.

Proof. By definition of $\mathcal{B}$, there exists at least one $e \in S^{1}$ such that $|F e|^{2}=m_{\mathcal{U}}(e)$. If there exists an $e$ such that $m_{\mathcal{U}}(e)=|F e|^{2}=\left|U_{i} e\right|^{2}>m_{\mathcal{U} \backslash\left\{U_{i}\right\}}(e)$ for some $i \in 1, \ldots, n$, then it follows from lemma 2.4 that $F=Q U_{i}$ with $Q \in \mathrm{SO}(2)$ and thus (i) follows from lemmas 2.6 and 2.5. Therefore, we may assume (relabelling the matrices if necessary) that there exists $2 \leqslant n \leqslant k$ such that

$$
|F e|^{2}=\left|U_{1} e\right|^{2}=\cdots=\left|U_{n} e\right|^{2}=m_{\mathcal{U}}(e)>\max \left\{\left|U_{i} e\right|^{2}: i=n+1, \ldots, k\right\} .
$$

Let $p, q \in\{1, \ldots, n\}, p \neq q$, be the indices with the properties stated in lemma 2.5. By lemma 2.1, there exist $Q \in \mathrm{SO}(2), \alpha \in \mathbb{R} \backslash\{0\}$ such that 
$Q U_{q}-U_{p}=\alpha U_{p} e \otimes e^{\perp}$. Note that $\alpha>0$. Indeed, by expansion and lemma 2.5 with $e_{\theta}=\left(1+\theta^{2}\right)^{-1 / 2}\left(e+\theta e^{\perp}\right)$, we obtain

$$
\left|U_{q} e_{\theta}\right|^{2}=\left|U_{p} e_{\theta}\right|^{2}+\frac{2 \theta \alpha}{1+\theta^{2}}\left|U_{p} e\right|^{2}+\mathcal{O}\left(\theta^{2}\right)<\left|U_{p} e_{\theta}\right|^{2} \quad \forall \theta \in\left(-\theta_{0}, 0\right),
$$

and this proves the asserted inequality. By lemma 2.3, $F=\tilde{Q}\left(U_{p}+s \alpha U_{p} e \otimes e^{\perp}\right)$ for some $\tilde{Q} \in \mathrm{SO}(2), s \in \mathbb{R}$, so that

$$
\left|F e_{\theta}\right|^{2}=\left|U_{p} e_{\theta}\right|^{2}+\frac{2 \theta \alpha s}{1+\theta^{2}}\left|U_{p} e\right|^{2}+\mathcal{O}\left(\theta^{2}\right) .
$$

Since $F \in \mathcal{B}$, we have $\left|F e_{\theta}\right|^{2} \leqslant m_{\mathcal{U}}\left(e_{\theta}\right)=\left|U_{p} e_{\theta}\right|^{2}$ for $-\theta_{0}<\theta<0$, and we conclude that $s \geqslant 0$. Similarly, $\left|F e_{\theta}\right|^{2} \leqslant\left|U_{q} e_{\theta}\right|^{2}$ for $0<\theta<\theta_{0}$ and therefore

$$
\left|U_{p} e_{\theta}\right|^{2}+\frac{2 \theta \alpha s}{1+\theta^{2}}\left|U_{p} e\right|^{2}+\mathcal{O}\left(\theta^{2}\right) \leqslant\left|U_{p} e_{\theta}\right|^{2}+\frac{2 \theta \alpha}{1+\theta^{2}}\left|U_{p} e\right|^{2}+\mathcal{O}\left(\theta^{2}\right),
$$

and we conclude that $s \leqslant 1$. This proves (i). Finally, part (ii) follows from the observation that

$$
\left\{F \in \mathbb{M}^{2 \times 2}: \operatorname{det} F=\delta, F^{\mathrm{T}} F \in \Gamma_{p, q}\left(e ; m_{\mathcal{U}}(e)\right)\right\} \subset\left(\mathrm{SO}(2) U_{p} \cup \mathrm{SO}(2) U_{q}\right)^{(1)},
$$

using the definition of $\mathcal{B}$.

We are now in a position to prove proposition 3.1 .

Proof of proposition 3.1. In view of lemma 3.2, it remains to show $\mathcal{A} \subset K^{(2)} \subset K^{\text {lc }}$. By lemma 3.3, we have $\mathcal{B} \subset K^{(1)} \subset K^{(2)}$. Assume now that $F \in \mathcal{A} \backslash \mathcal{B}$. Fix any $e \in S^{1}$ and let $F_{t}=F\left(I+t e \otimes e^{\perp}\right)$ and

$$
C(t)=F_{t}^{\mathrm{T}} F_{t}=F^{\mathrm{T}} F+t\left(F^{\mathrm{T}} F e \otimes e^{\perp}+e^{\perp} \otimes F^{\mathrm{T}} F e\right)+t^{2}|F e|^{2} e^{\perp} \otimes e^{\perp} .
$$

Since $F e \neq 0$, we conclude $|C(t)|^{2} \rightarrow \infty$ for $t \rightarrow \pm \infty$ and therefore

$$
\begin{aligned}
& t^{+}=\sup \left\{t>0:\left|F_{s} e\right|^{2}<m_{\mathcal{U}}(e) \forall e \in S^{1} \forall s \in[0, t]\right\}, \\
& t^{-}=\inf \left\{t<0:\left|F_{s} e\right|^{2}<m_{\mathcal{U}}(e) \forall e \in S^{1} \forall s \in[0, t]\right\}
\end{aligned}
$$

are well defined and $-\infty<t^{-}<0<t^{+}<\infty$. By construction, $F^{\mathrm{T}} F$ is contained in the arc connecting $C^{+}$and $C^{-}$on the curve $\Gamma\left(e ;|F e|^{2}\right)$. Let $V^{ \pm}$be the square root of $C^{ \pm}$. Then $F \in\left(\mathrm{SO}(2) V^{+} \cup \mathrm{SO}(2) V^{-}\right)^{(1)}$ and, since $V^{ \pm} \in \mathcal{B} \subset K^{(1)}$, we conclude $\mathcal{A} \subset K^{(2)}$. This proves the proposition.

The quasiconvex hull of two martensitic wells in two dimensions with equal determinant $\delta>0$ was first obtained by Ball and James [4]. We recover their result as a special case in proposition 3.1 .

EXAmple 3.4 (The two-well problem). Assume that $\operatorname{det} U_{1}=\operatorname{det} U_{2}=\delta>0$, $\mathrm{SO}(2) U_{1} \neq \mathrm{SO}(2) U_{2}$ and let $K=\mathrm{SO}(2) U_{1} \cup \mathrm{SO}(2) U_{2}$. Then there exist two vectors $e_{1}, e_{2}$ such that

$$
K^{\mathrm{qc}}=\left\{F \in \mathbb{M}^{2 \times 2}: \operatorname{det} F=\delta,\left|F e_{i}\right|^{2} \leqslant \max \left\{\left|U_{1} e_{i}\right|^{2},\left|U_{2} e_{i}\right|^{2}\right\}, i=1,2\right\} .
$$


It is easy to see that for $k=2$ there exist exactly two rank-one connections between the wells $\mathrm{SO}(2) U_{1}$ and $\mathrm{SO}(2) U_{2}$, i.e. there exist $Q_{i} \in \mathrm{SO}(2)$ and $a_{i}, e_{i} \in \mathbb{R}^{2}$ such that $Q_{i} U_{1}-U_{2}=a_{i} \otimes e_{i}^{\perp}$. Let $\mathcal{U}=\left\{U_{1}, U_{2}\right\}$ and

$$
\mathcal{A}=\left\{F \in \mathbb{M}^{2 \times 2}: \operatorname{det} F=\delta,\left|F e_{i}\right|^{2} \leqslant m_{\mathcal{U}}\left(e_{i}\right), i=1,2\right\} .
$$

We have to show that $F \in \mathcal{A}$ implies $|F e|^{2} \leqslant m_{\mathcal{U}}(e)$ for all $e \in S^{1}$. Assume the contrary. Then there exists an $e \in S^{1}$ such that $|F e|^{2}>m_{\mathcal{U}}(e)$. Assume first that $\left|F e_{1}\right|^{2}=m_{\mathcal{U}}\left(e_{1}\right)$ (the case that this equality holds for $e_{2}$ is similar). There exist $t_{0} \in \mathbb{R}$ and $Q_{1} \in \mathrm{SO}(2)$ such that $F-Q_{1} U_{1}=t_{0} Q_{1} U_{1} e_{1} \otimes e_{1}^{\perp}$. Let $F(t)=Q_{1} U_{1}+t Q_{1} U_{1} e_{1} \otimes e_{1}^{\perp}$. By assumption, there exist $t_{2} \in \mathbb{R}$ and $Q_{2} \in \mathrm{SO}(2)$ such that $F\left(t_{2}\right)=Q_{2} U_{2}$. Since $g(t)=\left|F(t) e_{2}\right|^{2}>0$ is a quadratic function with $g(0)=g\left(t_{2}\right) \geqslant g\left(t_{0}\right)$, we conclude $t_{2} \leqslant t_{0} \leqslant 0$ or $0 \leqslant t_{0} \leqslant t_{2}$. This shows that $F=\lambda Q_{1} U_{1}+(1-\lambda) Q_{2} U_{2}$ with $Q_{1}, Q_{2} \in \mathrm{SO}(2)$ and $\lambda \in[0,1]$. Thus

$$
m_{\mathcal{U}}(e)<|F e|^{2} \leqslant \lambda\left|U_{1} e\right|^{2}+(1-\lambda)\left|U_{2} e\right|^{2} \leqslant m_{\mathcal{U}}(e),
$$

and we conclude that $|F e|^{2}=m_{\mathcal{U}}(e)$; a contradiction. Thus we may assume that $\left|F e_{i}\right|<m_{\mathcal{U}}\left(e_{i}\right)$ for $i=1,2$. Let $F_{t}=F+t F e \otimes e^{\perp}$. Then $\operatorname{det} F_{t}=\delta$ and

$$
\left|F_{t} e_{i}\right|^{2}=\left|F e_{i}+t\left\langle e_{i}, e^{\perp}\right\rangle F e\right|^{2} .
$$

Since $e_{1}$ and $e_{2}$ are linearly independent, $\left\langle e_{i}, e^{\perp}\right\rangle \neq 0$ for at least one of the two indices and we may choose $s>0$ such that $\left|F_{s} e_{1}\right|^{2}=m_{\mathcal{U}}\left(e_{1}\right)$ and $\left|F_{s} e_{2}\right|^{2} \leqslant m_{\mathcal{U}}\left(e_{2}\right)$ (or vice versa). Clearly, $F_{s} \in \mathcal{A}$ and it follows as above that $\left|F_{s} e\right|^{2}=|F e|^{2}=m_{\mathcal{U}}(e)$; a contradiction. See figure 5 for a sketch of the set where $U_{1}$ and $U_{2}$ are diagonal. Conversely, any set $\tilde{K}$ on the hyperboloid $\left\{X=F^{\mathrm{T}} F: \operatorname{det} F=\delta\right\}$, which is bounded by two arcs of the form above, can be described by

$$
\tilde{K}=\left\{F^{\mathrm{T}} F: F \in\left(\mathrm{SO}(2) U_{1} \cup \mathrm{SO}(2) U_{2}\right)^{\mathrm{qc}}\right\} .
$$

We now turn to the proof theorem 1.1 (i), which says that the generalized convex hulls are always described by a finite number of vectors, as in example 3.4 . The next rather technical lemmas are the main ingredient in the proof. Let

$$
\tilde{\mathcal{U}}=\left\{U_{i} \in \mathcal{U}:\left|U_{i} \tilde{e}\right|^{2}>m_{\mathcal{U} \backslash\left\{U_{i}\right\}}(\tilde{e}) \text { for some } \tilde{e} \in S^{1}\right\} .
$$

Relabelling the matrices if necessary, we may assume that

$$
\tilde{\mathcal{U}}=\left\{U_{1}, \ldots, U_{n}\right\} .
$$

Lemma 3.5. Let $\mathcal{U}$ and $\tilde{\mathcal{U}}$ be defined as above. Then

$$
\left(\bigcup_{i=1}^{k} \mathrm{SO}(2) U_{i}\right)^{\mathrm{qc}}=\left(\bigcup_{i=1}^{n} \mathrm{SO}(2) U_{i}\right)^{\mathrm{qc}} .
$$

Proof. In view of proposition 3.1, we only have to show that $\left|U_{i} e\right|^{2} \leqslant m_{\tilde{\mathcal{u}}}(e)$ for all $e \in S^{1}$. It suffices to show this for $U_{k}$. Assume that there exists an $e \in S^{1}$ such that $\left|U_{k} e\right|^{2}>m_{\tilde{\mathcal{U}}}(e)$. Relabelling the matrices (if necessary), we may assume that there exists an $\ell \in\{n+1, \ldots, k\}$ such that $\left|U_{i} e\right|^{2} \geqslant\left|U_{k} e\right|^{2}>m_{\tilde{\mathcal{U}}}(e)$ for $i=\ell, \ldots, k$ and $\left|U_{i} e\right|^{2}<\left|U_{k} e\right|^{2}$ for $i=n+1, \ldots, \ell-1$. If $k=\ell$, then $\left|U_{k} e\right|^{2}>m_{\mathcal{U} \backslash\left\{U_{k}\right\}}$, contradicting 


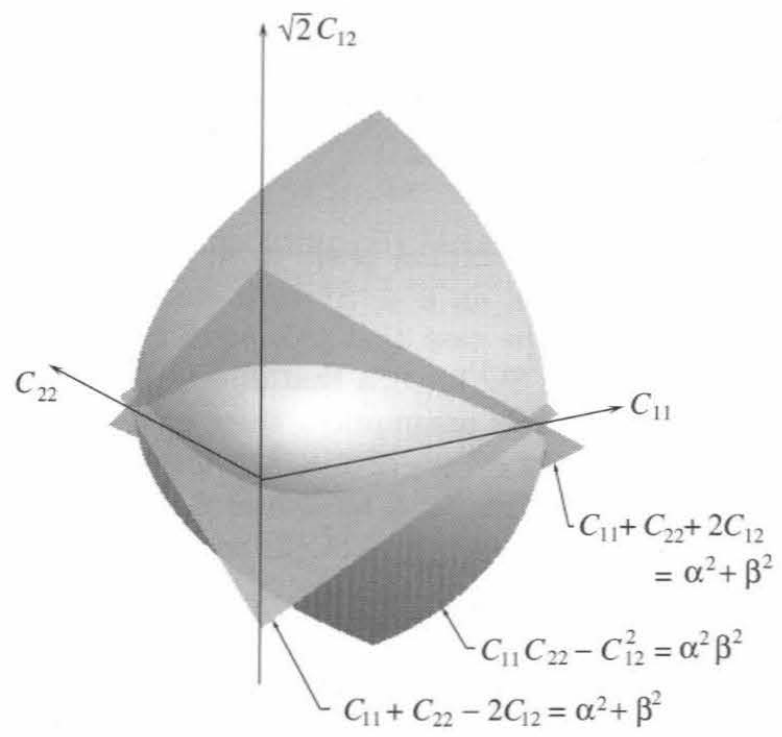

Figure 5. The set $\left(\mathrm{SO}(2) U_{1} \cup \mathrm{SO}(2) U_{2}\right)_{\mathrm{c}}^{\text {qc }}$ for the diagonal matrices $U_{1}=\operatorname{diag}(\alpha, \beta)$ and $U_{2}=\operatorname{diag}(\beta, \alpha)$.

$U_{k} \in \mathcal{U} \backslash \tilde{\mathcal{U}}$. We obtain the same contradiction if there exists an $i \in\{\ell, \ldots, k\}$ such that $\left|U_{i} e\right|^{2}>\max \left\{\left|U_{j} e\right|^{2}, j=\ell, \ldots, k, j \neq i\right\}$. Thus we may assume (relabelling again the matrices, if necessary) that $U_{\ell}, \ldots, U_{k} \in \mathcal{U} \backslash \tilde{\mathcal{U}}$ satisfy

$$
\left|U_{\ell} e\right|^{2}=\cdots=\left|U_{k} e\right|^{2}>m_{\left\{U_{1}, \ldots, U_{l-1}\right\}}(e)
$$

with $\ell<k$. In this situation, it follows from lemma 2.5 (i) that there exists a $p \in\{\ell, \ldots, k\}$ and an $e_{\theta} \in S^{1}$ such that

$$
\left|U_{p} e_{\theta}\right|^{2}>m_{\mathcal{U} \backslash\left\{U_{p}\right\}}\left(e_{\theta}\right),
$$

contradicting the assumption $U_{p} \in \mathcal{U} \backslash \tilde{\mathcal{U}}$. This proves the assertion of the lemma.

Lemma 3.6. Assume that $n \geqslant 2$. The set $\tilde{\mathcal{U}}$ has the following properties.

(i) If $e \in S^{1}$ and $U_{i}, U_{j} \in \tilde{\mathcal{U}}, i \neq j$, such that $\left|U_{i} e\right|^{2}=\left|U_{j} e\right|^{2}=m_{\mathcal{U}}(e)$, then $\left|U_{\ell} e\right|^{2}<m_{\mathcal{U}}(e)$ for all $\ell \in\{1, \ldots, n\} \backslash\{i, j\}$.

(ii) For all $U_{i} \in \tilde{\mathcal{U}}$, there exist exactly two matrices $U_{i_{1}}, U_{i_{2}} \in \tilde{\mathcal{U}}, i \notin\left\{i_{1}, i_{2}\right\}$, and exactly two non-parallel vectors $e_{1}, e_{2} \in S^{1}$ such that

$$
\left|U_{i} e_{j}\right|^{2}=\left|U_{i_{j}} e_{j}\right|^{2}=m_{\mathcal{U}}\left(e_{j}\right)
$$

for $j=1,2$ and $\Gamma_{i, i_{j}}\left(e_{j} ; m_{\mathcal{U}}\left(e_{j}\right)\right) \subset\left\{F^{\mathrm{T}} F: F \in \mathcal{B}\right\}$.

(iii) Assume that $\Gamma_{\ell}\left(e_{\ell} ; m_{\mathcal{U}}\left(e_{\ell}\right)\right)=\Gamma_{i_{\ell}, j_{\ell}}\left(e_{\ell} ; m_{\mathcal{U}}\left(e_{\ell}\right)\right), \ell=1,2$, are two of the arcs constructed in (ii) and let $\dot{\Gamma}_{\ell}=\Gamma_{\ell} \backslash\left\{U_{i_{\ell}}^{\mathrm{T}} U_{i_{\ell}}, U_{j_{\ell}}^{\mathrm{T}} U_{j_{\ell}}\right\}$. Then $\dot{\Gamma}_{1} \cap \dot{\Gamma}_{2}=\emptyset$. 
(iv) For each $F \in \mathcal{B}$, there exist $U_{i}, U_{j} \in \tilde{\mathcal{U}}, i \neq j$, and $e \in S^{1}$ such that

$$
\left|U_{i} e\right|^{2}=\left|U_{j} e\right|^{2}=|F e|^{2}=m_{\mathcal{U}}(e)
$$

and $F^{\mathrm{T}} F \in \Gamma_{i, j}\left(e ; m_{\mathcal{U}}(e)\right) \subset\left\{G^{\mathrm{T}} G: G \in \mathcal{B}\right\}$.

Proof. We first prove parts (i), (iii) and (iv), and then (ii).

(i) Assume that there are three distinct matrices $U_{i}, U_{j}, U_{\ell} \in \tilde{\mathcal{U}}$ such that

$$
\left|U_{i} e\right|^{2}=\left|U_{j} e\right|^{2}=\left|U_{\ell} e\right|^{2}=m_{\mathcal{U}}(e) .
$$

By lemma 2.5, there exists $\beta \in\{i, j, \ell$,$\} and Q_{\alpha}, Q_{\gamma} \in \mathrm{SO}(2), \lambda \in(0,1)$ such that $U_{\beta}=\lambda Q_{\alpha} U_{\alpha}+(1-\lambda) Q_{\gamma} U_{\gamma}$, where $\{\alpha, \beta, \gamma\}=\{i, j, \ell\}$. Since $U_{\beta} \in \tilde{\mathcal{U}}$, there exists $\tilde{e} \in S^{1}$ such that $\left|U_{\beta} \tilde{e}\right|^{2}=m_{\mathcal{U}}(\tilde{e})>m_{\mathcal{U} \backslash\left\{U_{\beta}\right\}}(\tilde{e})$. Then

$$
m_{\mathcal{U}}(\tilde{e})=\left|U_{\beta} \tilde{e}\right|^{2} \leqslant \lambda\left|U_{\alpha} \tilde{e}\right|^{2}+(1-\lambda)\left|U_{\gamma} \tilde{e}\right|^{2} \leqslant m_{\mathcal{U}}(\tilde{e}),
$$

and therefore $\left|U_{\alpha} \tilde{e}\right|^{2}=\left|U_{\gamma} \tilde{e}\right|^{2}=m_{\mathcal{U}}(\tilde{e})$. This contradicts the assumption and we conclude $U_{\beta} \notin \tilde{\mathcal{U}}$.

(iii) Assume that $F^{\mathrm{T}} F \in \stackrel{\circ}{\Gamma}_{1} \cap \dot{\Gamma}_{2}$. By construction, there exist $Q_{i}, Q_{j} \in \mathrm{SO}(2)$ and $\lambda \in(0,1)$ such that $\lambda Q_{i} U_{i_{2}}+(1-\lambda) Q_{j} U_{j_{2}}=F$. By assumption,

$$
m_{\mathcal{U}}\left(e_{1}\right)=\left|F e_{1}\right|^{2} \leqslant \lambda\left|U_{i_{2}} e_{1}\right|^{2}+(1-\lambda)\left|U_{j_{2}} e_{1}\right|^{2} \leqslant m_{\mathcal{U}}\left(e_{1}\right),
$$

and thus

$$
\left|U_{i_{1}} e_{1}\right|^{2}=\left|U_{j_{1}} e_{1}\right|^{2}=\left|U_{i_{2}} e_{1}\right|^{2}=\left|U_{j_{2}} e_{1}\right|^{2}=m_{\mathcal{U}}\left(e_{1}\right) .
$$

If $\left\{i_{1}, j_{1}\right\} \neq\left\{i_{2}, j_{2}\right\}$, this contradicts (i) and we obtain the assertion.

Otherwise, we conclude by lemma 2.2 that $e_{1}$ and $e_{2}$ are not parallel and that there exist $a_{1}, a_{2} \in \mathbb{R}^{2}, Q_{1}, Q_{2} \in \mathrm{SO}(2)$ such that

$$
Q_{1} U_{i}-U_{j}=a_{1} \otimes e_{1}^{\perp}, \quad Q_{2} U_{i}-U_{j}=a_{2} \otimes e_{2}^{\perp},
$$

where we write $U_{i}$ and $U_{j}$ instead of $U_{i_{\ell}}$ and $U_{j_{\ell}}$. Let $F_{\lambda}=U_{j}+\lambda a_{1} \otimes e_{1}^{\perp}$. In order to show that the arcs $\Gamma_{i, j}\left(e_{1}, m_{\mathcal{U}}\left(e_{1}\right)\right)$ and $\Gamma_{i, j}\left(e_{2}, m_{\mathcal{U}}\left(e_{2}\right)\right)$ do not intersect, it suffices to show that $\left|F_{\lambda} e_{2}\right|^{2}<m_{\mathcal{U}}\left(e_{2}\right)$ for $\lambda \in(0,1)$. For $\lambda=1$, we obtain

$$
\left|Q_{1} U_{i} e_{2}\right|^{2}=\left|U_{j} e_{2}\right|^{2}+2\left\langle e_{1}^{\perp}, e_{2}\right\rangle\left\langle U_{j} e_{2}, a_{1}\right\rangle+\left\langle e_{1}^{\perp}, e_{2}\right\rangle^{2}\left|a_{1}\right|^{2}=0,
$$

and thus, by assumption,

$$
2\left\langle e_{1}^{\perp}, e_{2}\right\rangle\left\langle U_{j} e_{2}, a_{1}\right\rangle+\left\langle e_{1}^{\perp}, e_{2}\right\rangle^{2}\left|a_{1}\right|^{2}=0 .
$$

Therefore, $\alpha=2\left\langle e_{1}^{\perp}, e_{2}\right\rangle\left\langle U_{j} e_{2}, a_{1}\right\rangle<0$ and $\beta=\left\langle e_{1}^{\perp}, e_{2}\right\rangle^{2}\left|a_{1}\right|^{2}>0$ (note that $\left\langle e_{1}^{\perp}, e_{2}\right\rangle \neq 0$, since $e_{1}$ and $e_{2}$ are not parallel). Since $\left|F_{\lambda} e_{2}\right|^{2}<m_{\mathcal{U}}\left(e_{2}\right)$ if and only if $\lambda \alpha+\lambda^{2} \beta=\lambda(1-\lambda) \alpha<0$, we obtain the assertion.

(iv) This follows from lemma 3.3 .

(ii) This is easy for $n=2$ since there are exactly two rank-one connections between the wells. Thus we may assume that $n \geqslant 3$. Fix $U_{i}$. By lemma 2.6 combined with lemma 2.5 , there exists at least one $e_{1} \in S^{1}$ and $U_{j} \in \tilde{\mathcal{U}}, i \neq j$, such that $\left|U_{i} e_{1}\right|^{2}=\left|U_{j} e_{1}\right|^{2}=m_{\mathcal{U}}\left(e_{1}\right)$. In view of step 1 , we obtain $\left|U_{\ell} e_{1}\right|^{2}<m_{\mathcal{U}}\left(e_{1}\right)$ for $\ell \notin\{i, j\}$ and it follows from lemma 2.5 that the assumptions (ii) and (iii) in lemma 2.7 are satisfied for some $\varepsilon>0$. We conclude that there exist at least two 
linearly independent vectors $e_{1}, e_{2} \in S^{1}$ such that $\left|U_{i} e_{1}\right|^{2}=\left|U_{j} e_{1}\right|^{2}=m_{\mathcal{U}}\left(e_{1}\right)$ and $\left|U_{i} e_{2}\right|^{2}=\left|U_{\ell} e_{2}\right|^{2}=m_{\mathcal{U}}\left(e_{2}\right)$ with $U_{j}, U_{\ell} \in \tilde{\mathcal{U}}$ and $\ell \neq i$.

Assume now that $\left|U_{i_{j}} e_{j}\right|^{2}=\left|U_{i} e_{j}\right|^{2}=m_{\mathcal{U}}\left(e_{j}\right)$ for $j=1,2,3$, where no two of the vectors $e_{j}$ are parallel and $i \notin\left\{i_{1}, i_{2}, i_{3}\right\}$. If $i_{1}=i_{2}=i_{3}$, then it is easy to see that $U_{i}=Q U_{i_{1}}$ with $Q \in \mathrm{SO}(2)$, violating the general assumptions on $\tilde{\mathcal{U}}$. Thus we may assume that $i_{1} \neq i_{2}$. If $i_{1} \neq i_{2}=i_{3}$, then we define $\mathcal{V}=\left\{U_{i_{2}}, U_{i}\right\}$ and

$$
\mathcal{A}=\left\{F \in \mathbb{M}^{2 \times 2}: \operatorname{det} F=\delta,\left|F e_{j}\right|^{2} \leqslant m_{\mathcal{V}}\left(e_{i_{j}}\right), j=2,3\right\} .
$$

It follows from (i) that $U_{i_{1}} \in \mathcal{A}$, and we conclude with the same arguments as in example 3.4 that

$$
U_{i_{1}} \in\left(\mathrm{SO}(2) U_{i} \cup \mathrm{SO}(2) U_{i_{2}}\right)^{\mathrm{qc}} .
$$

By definition of $\tilde{\mathcal{U}}$, there exists an $\tilde{e} \in S^{1}$ such that $\left|U_{i_{1}} \tilde{e}\right|^{2}=m_{\mathcal{U}}(\tilde{e})>m_{\mathcal{U} \backslash U_{i_{1}}}(\tilde{e})$. However, by example 3.4 ,

$$
m_{\mathcal{U}}(\tilde{e})=\left|U_{i_{1}} \tilde{e}\right|^{2} \leqslant \max \left\{\left|U_{i} \tilde{e}\right|^{2},\left|U_{i_{2}} \tilde{e}\right|^{2}\right\} \leqslant m_{\mathcal{U}}(\tilde{e}) .
$$

This is a contradiction. Finally, assume $i_{j} \neq i_{\ell}$ for $j \neq \ell$. The curves $\Gamma\left(e_{j} ;\left|U_{i} e_{j}\right|^{2}\right)$ are the boundary of the regions $\left|U_{i} e_{j}\right|^{2}>m_{\mathcal{U}}\left(e_{j}\right)$ and $\left|U_{i} e_{j}\right|^{2}<m_{\mathcal{U}}\left(e_{j}\right)$, and using the ideas in the proof of (iii), we see that they intersect only at $U_{i}$. Thus each of these regions consists of just one connected component. Consider now the curve $\Gamma\left(e_{1} ; m_{\mathcal{U}}\left(e_{1}\right)\right)$. Then $U_{i_{2}}$ and $U_{i_{3}}$ must lie in the connected component $\left\{C=F^{\mathrm{T}} F: \operatorname{det} C=\delta^{2},\left|F e_{1}\right|^{2}<m_{\mathcal{U}}\left(e_{1}\right)\right\}$. Assume that the angle between the curves $\Gamma\left(e_{1} ; m_{\mathcal{U}}\left(e_{1}\right)\right)$ and $\Gamma\left(e_{2} ; m_{\mathcal{U}}\left(e_{2}\right)\right)$ is smaller than the angle between $\Gamma\left(e_{1} ; m_{\mathcal{U}}\left(e_{1}\right)\right)$ and $\Gamma\left(e_{3} ; m_{\mathcal{U}}\left(e_{3}\right)\right)$. Since $\left|U_{i} e_{1}\right|^{2}=\left|U_{i_{2}} e_{2}\right|^{2}=m_{\mathcal{U}}\left(e_{2}\right)$, we conclude $\left|U_{i_{1}} e_{2}\right|^{2}<m_{\mathcal{U}}\left(e_{2}\right)$ and thus $\left|U_{i_{3}} e_{2}\right|^{2}>m_{\mathcal{U}}\left(e_{2}\right)$; a contradiction. This proves assertion (ii) of the lemma.

With this information at hand, we can prove theorem 1.1 (i).

Proof of theorem 1.1 (i). Consider the graph $\mathcal{G}=\mathcal{G}(\mathcal{N}, \mathcal{E})$, where $\mathcal{N}=\tilde{\mathcal{U}}$ is the set of nodes and $\mathcal{E}$ is the set of edges which contains an edge connecting $U_{i}$ and $U_{j}$ if and only if there exists an $\operatorname{arc} \Gamma_{i, j}\left(e_{i j} ; m\left(e_{i j}\right)\right)$ with the properties in lemma 3.6. Thus there is a one-to-one correspondence of $\operatorname{arcs}$ in $\mathcal{B}$ and edges in $\mathcal{E}$ and it follows from lemma 3.6 that $\mathcal{G}$ is a graph of degree two (i.e. each node is contained in exactly two edges). It is easy to see that $\mathcal{G}$ must consist of disjoint cycles. By lemma 3.6, the $\operatorname{arcs} \Gamma_{i j}$ corresponding to the edges in the cycles do not intersect and therefore each of these cycles can be interpreted as a closed curve on the hyperboloid $\left\{\operatorname{det} C=\delta^{2}\right\} \subset \mathbb{R}^{3}$. It is easy to see that the set $\mathcal{A}$ is connected and therefore $\mathcal{G}$ must consist of a single cycle. It follows that $\mathcal{E}$ contains exactly $n$ edges. Let $\mathcal{E}_{n}$ be the set of normals $e_{i j}$ which define the arcs $\Gamma_{i j}$ corresponding to the edges in $\mathcal{E}$. By lemma $3.6, \mathcal{B}$ is the union of these arcs and therefore $K^{\mathrm{qc}}$ is defined by $k$ inequalities. This proves the assertion of the theorem.

EXAmple 3.7 (The four-well problem). Assume that $a, b, c>0, a>b, a b-c^{2}>0$ and define

$$
U_{1}=\left(\begin{array}{ll}
a & c \\
c & b
\end{array}\right), \quad U_{2}=\left(\begin{array}{ll}
b & c \\
c & a
\end{array}\right), \quad U_{3}=\left(\begin{array}{cc}
a & -c \\
-c & b
\end{array}\right), \quad U_{4}=\left(\begin{array}{cc}
b & -c \\
-c & a
\end{array}\right) .
$$


Let $\mathcal{U}=\left\{U_{1}, U_{2}, U_{3}, U_{4}\right\}$. Then

$$
K^{\mathrm{qc}}=\left\{F \in \mathbb{M}^{2 \times 2}: \operatorname{det} F=\delta,|F e|^{2} \leqslant m_{\mathcal{U}}(e) \forall e \in \mathcal{E}_{4}\right\},
$$

where

$$
\mathcal{E}_{4}=\left\{\frac{1}{\sqrt{ } 2}\left(\begin{array}{l}
1 \\
1
\end{array}\right), \frac{1}{\sqrt{ } 2}\left(\begin{array}{c}
1 \\
-1
\end{array}\right),\left(\begin{array}{l}
1 \\
0
\end{array}\right),\left(\begin{array}{l}
0 \\
1
\end{array}\right)\right\} .
$$

See figures 1 and 2 for a sketch of the set $K_{\mathrm{c}}^{\mathrm{qc}}=\left\{F^{\mathrm{T}} F: F \in K^{\mathrm{qc}}\right\}$ and the rank-one connections defining the boundaries on the manifold $\left\{\operatorname{det} C=\left(\operatorname{det} U_{1}\right)^{2}\right\}$.

We finally prove theorem 1.2 .

Proof of theorem 1.2. Let

$$
\mathcal{A}=\left\{F \in \mathbb{M}^{3 \times 3}: \operatorname{det} F=\delta, F^{\mathrm{T}} F v=\mu^{2} v,|F e|^{2} \leqslant \max _{i=1, \ldots, k}\left|U_{i} e\right|^{2} \forall e \in S^{2}\right\} .
$$

We first show that $K^{\mathrm{pc}} \subset \mathcal{A}$ by constructing a polyconvex function $\Phi$ which vanishes on $\mathcal{A}$ and is positive elsewhere. This generalizes a construction by Ball and James for the two-well problem. For $\nu \in S^{2}$, let $g_{\nu}: \mathbb{M}^{3 \times 3} \rightarrow \mathbb{R}$ be defined by

$$
g_{\nu}(X)=\left(|X \nu|^{2}-m_{\mathcal{U}}(\nu)\right)_{+},
$$

and let

$$
\Phi(X)=(\operatorname{det} X-\delta)^{2}+\sup _{\nu \in S^{2}} g_{\nu}(X)+\left(|X v|^{2}-\mu^{2}\right)_{+}+\left(|\operatorname{cof} X v|^{2}-\frac{\delta^{2}}{\mu^{2}}\right)_{+} .
$$

We have to show that $\Phi(F)=0$ implies $F^{\mathrm{T}} F v=\mu^{2} v$. Since $\operatorname{cof} F=(\operatorname{det} F) F^{-\mathrm{T}}$, it follows from $\Phi(F)=0$ that

$$
|F v|^{2} \leqslant \mu^{2}
$$

and

$$
|\operatorname{cof} F v|^{2} \leqslant \frac{\delta^{2}}{\mu^{2}} \Longleftrightarrow\left|F^{-\mathrm{T}} v\right|^{2} \leqslant \frac{1}{\mu^{2}}
$$

Then

$$
\begin{aligned}
\left|\left(\frac{1}{\mu} F\right)\left(v-\mu^{2} F^{-1} F^{-\mathrm{T}} v\right)\right| & =\left|\frac{1}{\mu} F v-\mu F^{-\mathrm{T}} v\right|^{2} \\
& =\frac{1}{\mu^{2}}|F v|^{2}+\mu^{2}\left|F^{-\mathrm{T}} v\right|^{2}-2 \leqslant 0,
\end{aligned}
$$

and since $\operatorname{det} F=\delta>0$, we conclude $v-\mu^{2} F^{-1} F^{-\mathrm{T}} v=0$. This implies the assertion.

We now show that $\mathcal{A} \subset K^{(2)}$. We will reduce the necessary constructions to the two-dimensional situation in theorem 1.1. Let $F \in \mathcal{A}$. By the polar decomposition theorem, we have $F=R U_{0}$, with $R \in \mathrm{SO}(3)$ and $U_{0}$ symmetric and positivedefinite. Since $F^{\mathrm{T}} F v=U_{0}^{2} v=\mu^{2} v$, we conclude $U_{0} v=\mu v$. Thus the matrices $U_{i}$, $i=0, \ldots, k$, have $\mu$ as common eigenvalue with corresponding eigenvector $v$. Choose an orthonormal basis $\left\{v_{1}, v_{2}, v_{3}=v\right\}$ and let $Q$ be the rotation with columns $v_{i}$. Then

$$
Q^{\mathrm{T}} U_{i} Q=\left(\begin{array}{cc}
\hat{U}_{i} & 0 \\
0 & \mu
\end{array}\right)
$$


with $\hat{U}_{i} \in \mathbb{M}_{\text {sym }}^{2 \times 2}$, $\operatorname{det} \hat{U}_{i}=\delta / \mu$. Let $\hat{\mathcal{U}}=\left\{\hat{U}_{1}, \ldots, \hat{U}_{k}\right\}$. Now define $\hat{\pi}: \mathbb{R}^{3} \rightarrow \mathbb{R}^{2}$ by $\hat{\pi}(u)=\left(u_{1}, u_{2}\right)$ and $\pi_{3}: \mathbb{R}^{3} \rightarrow \mathbb{R}$ by $\pi_{3}(u)=u_{3}$ for $u \in \mathbb{R}^{3}$. For $e \in S^{2}$, we have

$$
\begin{aligned}
|F e|^{2} & =\left|R U_{0} e\right|^{2} \\
& =\left|Q^{\mathrm{T}} U_{0} Q Q^{\mathrm{T}} e\right|^{2} \\
& =\left|\hat{U}_{0} \hat{\pi}\left(Q^{\mathrm{T}} e\right)\right|^{2}+\mu^{2}\left|\pi_{3}\left(Q^{\mathrm{T}} e\right)\right|^{2} \\
& \leqslant m_{\mathcal{U}}(e) \\
& =\max _{i=1, \ldots, k}\left|\hat{U}_{i} \hat{\pi}\left(Q^{\mathrm{T}} e\right)\right|^{2}+\mu^{2}\left|\pi_{3}\left(Q^{\mathrm{T}} e\right)\right|^{2} .
\end{aligned}
$$

If we choose $e$ such that $\hat{\pi}\left(Q^{\mathrm{T}} e\right) \in S^{1}$, then we obtain

$$
\left|\hat{U}_{0} e\right|^{2} \leqslant m_{\hat{\mathcal{U}}}(e) \quad \forall e \in S^{1} .
$$

It follows from theorem 1.1 that

$$
\hat{U}_{0} \in\left(\mathrm{SO}(2) \hat{U}_{1} \cup \cdots \cup \mathrm{SO}(2) \hat{U}_{k}\right)^{(2)} .
$$

Since $K$ is invariant under multiplication with elements in $\mathrm{SO}(3)$ from the left, and since $\operatorname{rank}(A-B)=1$ if and only if $\operatorname{rank}\left(Q(A-B) Q^{\mathrm{T}}\right)=1$, it follows that $F \in K^{(2)}$.

Finally, the reduction from an infinite to a finite number of inequalities in the definition of the hulls follows as in the proof of theorem 1.1(i).

\section{The quasiconvex hull of $\mathrm{O}(2) U_{1} \cup \cdots \cup \mathrm{O}(2) U_{k}$}

In this section we prove theorem 1.1(ii). We split the proof into a series of lemmas. As before, let $\mathcal{U}=\left\{U_{1}, \ldots, U_{k}\right\}$ and

$$
\mathcal{A}=\left\{F \in \mathbb{M}^{2 \times 2}:|\operatorname{det} F| \leqslant \delta,|F e|^{2} \leqslant m_{\mathcal{U}}(e) \forall e \in S^{1}\right\} .
$$

We will show that $K^{\mathrm{pc}} \subset \mathcal{A} \subset K^{(3)}$. This proves the theorem, since, by (2.2), $K^{\text {lc }} \subset K^{\text {pc }}$. We prove first the inclusion $K^{\text {pc }} \subset \mathcal{A}$ by constructing a polyconvex function which vanishes exactly on $\mathcal{A}$ and is positive elsewhere.

Lemma 4.1. Suppose the assumptions of theorem 1.1 hold and $\mathcal{A}$ is defined by (4.1). Then $K^{\mathrm{pc}} \subset \mathcal{A}$.

Proof. The proof is similar to lemma 3.2. Let

$$
\Phi(X)=(|\operatorname{det} X|-\delta)_{+}+\sup _{\nu \in S^{1}} g_{\nu}(X) .
$$

Since $t \mapsto(|t|-\delta)_{+}$is a convex function, $\Phi$ is a polyconvex function that vanishes on $\mathcal{A}$ and is positive elsewhere. The assertion follows now from the definition of the polyconvex hull.

The inclusion $\mathcal{A} \subset K^{(3)}$ requires some more work. We prove first two auxiliary results. 
Lemma 4.2. If $F, G \in \mathbb{M}^{2 \times 2}$ satisfy

$$
F^{\mathrm{T}} F=G^{\mathrm{T}} G-\alpha G^{\mathrm{T}} e \otimes G^{\mathrm{T}} e
$$

for some $\alpha \in[0,1]$ and some $e \in S^{1}$, then

$$
F \in(\mathrm{O}(2) G)^{(1)} .
$$

Proof. Since $\alpha \in[0,1]$, there exists $\lambda \in[0,1]$ such that $\alpha=4 \lambda(1-\lambda)$, so that

$$
F^{\mathrm{T}} F=G^{\mathrm{T}} G-4 \lambda(1-\lambda) G^{\mathrm{T}} e \otimes G^{\mathrm{T}} e=\left(G-2 \lambda e \otimes G^{\mathrm{T}} e\right)^{\mathrm{T}}\left(G-2 \lambda e \otimes G^{\mathrm{T}} e\right) .
$$

Therefore, $|F v|^{2}=\left|\left(G-2 \lambda e \otimes G^{\mathrm{T}} e\right) v\right|^{2}$ for all $v \in S^{1}$ and we conclude that there exists $\tilde{Q} \in \mathrm{O}(2)$ such that

$$
F=\tilde{Q}\left(G-2 \lambda e \otimes G^{\mathrm{T}} e\right) .
$$

If we define $Q=I-2 e \otimes e \in \mathrm{O}(2)$, then

$$
F=\tilde{Q}(\lambda Q G+(1-\lambda) G) \text { and } Q G-G=-2 e \otimes G^{\mathrm{T}} e,
$$

and this proves the lemma.

For the statement of the next lemma, it is useful to introduce some notation. Let

$$
\begin{aligned}
\mathcal{B} & =\left\{F:|\operatorname{det} F| \leqslant \delta,|F e|^{2} \leqslant m_{\mathcal{U}}(e) \forall e \in S_{1}, \exists \tilde{e}:|F \tilde{e}|^{2}=m_{\mathcal{U}}(\tilde{e})\right\}, \\
\mathcal{B}_{\alpha} & =\mathcal{B} \cap\{F: \operatorname{det} F=\alpha\} \quad \text { for } \alpha \in[-\delta, \delta], \\
\mathcal{A}_{\alpha} & =\mathcal{A} \cap\{F: \operatorname{det} F=\alpha\} \quad \text { for } \alpha \in[-\delta, \delta] .
\end{aligned}
$$

Lemma 4.3. Assume that $F \in \mathcal{B}$, with $|\operatorname{det} F|<\delta$. Then one of the following alternatives holds.

(i) There exists a unique (up to the sign) $e \in S^{1}$ such that

$$
|F e|^{2}=\left|U_{i} e\right|^{2}>m_{\mathcal{U} \backslash\left\{U_{i}\right\}}(e) \quad \text { and } F^{\mathrm{T}} F=U_{i}^{\mathrm{T}} U_{i}-\alpha e^{\perp} \otimes e^{\perp},
$$

where $\alpha \in\left(0,\left|U_{i}^{-\mathrm{T}} e^{\perp}\right|^{-2}\right]$. Equivalently,

$$
F^{\mathrm{T}} F=U_{i}^{\mathrm{T}} U_{i}-\tilde{\alpha} U_{i}^{\mathrm{T}} \tilde{e} \otimes U_{i}^{\mathrm{T}} \tilde{e}, \quad \text { with } \tilde{e}=U_{i}^{-\mathrm{T}} e^{\perp} /\left|U_{i}^{-\mathrm{T}} e^{\perp}\right| \in S^{1}
$$

and $\tilde{\alpha}=\alpha\left|U_{i}^{-\mathrm{T}} e^{\perp}\right|^{2} \in(0,1]$.

(ii) There exists a unique (up to the sign) $e \in S^{1}$ and $a G \in \mathcal{B}_{\delta}$ such that (relabelling the matrices if necessary)

$$
|F e|^{2}=|G e|^{2}=\left|U_{1} e\right|^{2}=\cdots=\left|U_{n} e\right|^{2}>m_{\mathcal{U} \backslash\left\{U_{1}, \ldots, U_{n}\right\}}(e),
$$

with $n \geqslant 2$. Moreover, $F^{\mathrm{T}} F=G^{\mathrm{T}} G-\alpha e^{\perp} \otimes e^{\perp}$ with $\alpha \in\left(0,\left|G^{-\mathrm{T}} e^{\perp}\right|^{-2}\right]$ or, equivalently,

$$
F^{\mathrm{T}} F=G^{\mathrm{T}} G-\tilde{\alpha} G^{\mathrm{T}} \tilde{e} \otimes G^{\mathrm{T}} \tilde{e}, \quad \text { with } \tilde{e}=G^{-\mathrm{T}} e^{\perp} /\left|G^{-\mathrm{T}} e^{\perp}\right| \in S^{1}
$$

and $\tilde{\alpha}=\alpha\left|G^{-\mathrm{T}} e\right|^{2} \in(0,1]$. 
Proof. By definition of $\mathcal{B}$ there exists at least one $e \in S^{1}$ such that $|F e|^{2}=m_{\mathcal{U}}(e)$. Assume first that there exist $e \in S^{1}$ and $U_{i} \in \mathcal{U}$ such that

$$
m_{\mathcal{U}}(e)=|F e|^{2}=\left|U_{i} e\right|^{2}>m_{\mathcal{U} \backslash\left\{U_{i}\right\}^{(e)}}
$$

for some $i \in 1, \ldots, k$. It follows from lemma 2.4 that $F^{\mathrm{T}} F=U_{i}^{2}-\alpha e^{\perp} \otimes e^{\perp}$ for some $\alpha \geqslant 0$. Since

$$
0 \leqslant(\operatorname{det} F)^{2}=\left(\operatorname{det} U_{i}\right)^{2}\left(1-\alpha\left|U_{i}^{-\mathrm{T}} e^{\perp}\right|^{2}\right)<\delta^{2},
$$

we conclude that $\alpha \in\left(0,\left|U_{i}^{-\mathrm{T}} e^{\perp}\right|^{-2}\right]$. The uniqueness of $e$ follows now from $\alpha>0$ and $|F \tilde{e}|^{2}=\left|U_{i} \tilde{e}\right|^{2}-\alpha\left(\tilde{e}, e^{\perp}\right)^{2}$. This proves (i).

Assume now that there exists (relabelling the matrices if necessary) $n \in\{2, \ldots, k\}$ such that

$$
\left|U_{1} e\right|^{2}=\ldots=\left|U_{n} e\right|^{2}=m_{\mathcal{U}}(e)>m_{\mathcal{U} \backslash\left\{U_{1}, \ldots, U_{n}\right\}}(e) .
$$

By lemma 2.5, we find $p, q \in\{1, \ldots, n\}, p \neq q$, such that

(a) $m_{\mathcal{U}}\left(e_{\theta}\right)=\left|U_{p} e_{\theta}\right|^{2}>m_{\mathcal{U} \backslash\left\{U_{p}\right\}}\left(e_{\theta}\right)$ for $-\theta_{0}<\theta<0$,

(b) $m_{\mathcal{U}}\left(e_{\theta}\right)=\left|U_{q} e_{\theta}\right|^{2}>m_{\mathcal{U} \backslash\left\{U_{q}\right\}}\left(e_{\theta}\right)$ for $0<\theta<\theta_{0}$,

where $e_{\theta}={\sqrt{1+\theta^{2}}}^{-1}\left(e+\theta e^{\perp}\right)$. According to lemma 2.1, there exist $a, b \in \mathbb{R}^{2}$ with

$$
F^{\mathrm{T}} F=U_{p}^{2}+a \otimes e^{\perp}+e^{\perp} \otimes a, \quad F^{\mathrm{T}} F=U_{q}^{2}+b \otimes e^{\perp}+e^{\perp} \otimes b .
$$

It follows from (a) above that

$$
\left|F e_{\theta}\right|^{2}=\left|U_{p} e_{\theta}\right|^{2}+2 \theta\left(\langle a, e\rangle+\theta\left\langle a, e^{\perp}\right\rangle\right) \leqslant m_{\mathcal{U}}\left(e_{\theta}\right)=\left|U_{p} e_{\theta}\right|^{2}
$$

for all $\theta \in\left(-\theta_{0}, 0\right)$. We conclude that $\langle a, e\rangle \geqslant 0$. Choosing $\theta \in\left(0, \theta_{0}\right)$ and observing (b), we deduce that $\langle b, e\rangle \leqslant 0$. Therefore, there exists $\lambda \in[0,1], t \in \mathbb{R}$ such that

$$
\lambda a+(1-\lambda) b=t e^{\perp}
$$

(we allow $t=0$ if $a$ and $b$ are linearly dependent; in this case, we have that $\left.\operatorname{det}\left(F^{\mathrm{T}} F\right)=\delta^{2}\right)$. We now define, for $\mu \in[0,1]$,

$$
\begin{aligned}
C_{\lambda, \mu} & =\lambda\left(\mu U_{p}^{2}+(1-\mu) F^{\mathrm{T}} F\right)+(1-\lambda)\left(\mu U_{q}^{2}+(1-\mu) F^{\mathrm{T}} F\right) \\
& =F^{\mathrm{T}} F-2 t \mu e^{\perp} \otimes e^{\perp} .
\end{aligned}
$$

By construction,

$$
\operatorname{det} C_{\lambda, 0}=\operatorname{det} F^{\mathrm{T}} F \leqslant \delta^{2},
$$

and a simple calculation shows that

$$
\begin{aligned}
\operatorname{det} C_{\lambda, 1} & =\operatorname{det}\left(\lambda U_{p}^{2}+(1-\lambda) U_{q}^{2}\right) \\
& =\lambda \operatorname{det} U_{p}^{2}+(1-\lambda) \operatorname{det} U_{q}^{2}-\lambda(1-\lambda) \operatorname{det}\left(U_{p}^{2}-U_{q}^{2}\right) \\
& \geqslant \delta^{2},
\end{aligned}
$$

since, according to lemma $2.1, \operatorname{det}\left(U_{p}^{2}-U_{q}^{2}\right) \leqslant 0$. Therefore, there exists $\tilde{\mu}$ such that

$$
\operatorname{det} C_{\lambda, \tilde{\mu}}=\delta^{2} .
$$


By construction, $\left\langle e, C_{\lambda, \tilde{\mu}} e\right\rangle=m(e)$ and, moreover, $\left\langle v, C_{\lambda, \tilde{\mu}} v\right\rangle \leqslant m(v)$ for all $v \in S^{1}$, since $C_{\lambda, \tilde{\mu}}$ is a convex combination of three matrices which satisfy these inequalities. Therefore, we conclude that there exists $G \in \mathcal{B}_{\delta}$ such that $G^{\mathrm{T}} G=C_{\lambda, \tilde{\mu}}$ and

$$
F^{\mathrm{T}} F=G^{\mathrm{T}} G+2 t \tilde{\mu} e^{\perp} \otimes e^{\perp} .
$$

In particular, $|G e|^{2}=|F e|^{2}=m_{\mathcal{U}}(e)$. Let $\tilde{e}=G^{-\mathrm{T}} e^{\perp}$. Finally, notice that $\operatorname{det} F^{\mathrm{T}} F<\delta^{2}$ implies that $2 t \tilde{\mu}<0$ and this proves the uniqueness of $e$.

Proof of theorem 1.1 (ii). In view of lemma 4.1, it remains to show that $\mathcal{A} \subset K^{(3)}$. By lemma 3.3,

$$
\mathcal{B}_{\delta} \subset\left(\mathrm{SO}(2) U_{1} \cup \cdots \cup \mathrm{SO}(2) U_{k}\right)^{(1)} \subset K^{(1)},
$$

and since $\mathcal{B}_{-\delta}=Q \mathcal{B}_{\delta}$ for any $Q \in \mathrm{O}(2) \backslash \mathrm{SO}(2)$, we conclude that $\mathcal{B}_{ \pm \delta} \subset K^{(1)}$. Combining this with lemmas 4.3 and 4.2 , it follows that $\mathcal{B} \subset K^{(2)}$. Now, for any $F \in \mathcal{B}_{\alpha}, \alpha \in[-\delta, \delta] \backslash\{0\}$, we use the arguments in the proof of theorem 1.1 (i) to construct two rank-one connected matrices on the manifold $\{\operatorname{det} X=\alpha\}$ such that $F$ is contained in the rank-one segment connecting these two matrices. Thus $\mathcal{A} \subset K^{(3)}$. Finally, consider any $F \in \mathcal{A}_{0}$. Clearly, $F=Q(\beta e \otimes e)$ for some $Q \in \mathrm{O}(2)$, $\beta \in \mathbb{R}$ and $e \in S^{1}$ and, by definition,

$$
|F v|^{2}=\beta^{2}\langle e, v\rangle^{2} \leqslant m_{\mathcal{U}}(v) \quad \forall v \in S^{1} .
$$

By continuity, there exists $\gamma^{2} \geqslant \beta^{2}$ such that $G=\gamma e \otimes e \in \mathcal{B} \subset K^{(2)}$. Therefore, $F^{\mathrm{T}} F=G^{\mathrm{T}} G-\alpha G^{\mathrm{T}} e \otimes G^{\mathrm{T}} e$ with $\alpha=\left(\gamma^{2}-\beta^{2}\right) / \gamma^{2} \in[0,1]$, and consequently $F \in(\mathrm{O}(2) G)^{(1)} \subset K^{(3)}$ by lemma 4.2. This implies the assertion of the theorem.

EXAMPLE 4.4 (The two-well problem). Assume that $\mathcal{U}=\left\{U_{1}, U_{2}\right\}$, where $U_{1}, U_{2} \in$ $\mathbb{M}^{2 \times 2}$ with $\operatorname{det} U_{1}=\operatorname{det} U_{2}=\delta>0$, and that $\mathrm{O}(2) U_{1} \neq O(2) U_{2}$. Let $K=\mathrm{O}(2) U_{1} \cup$ $\mathrm{O}(2) U_{2}$. Then

$$
K^{\mathrm{qc}}=\left\{F \in \mathbb{M}^{2 \times 2}:|\operatorname{det} F| \leqslant \delta,|F e|^{2} \leqslant m_{\mathcal{U}}(e) \forall e \in S^{1}\right\} .
$$

The set $K_{\mathrm{c}}^{\mathrm{qc}}=\left\{F^{\mathrm{T}} F: F \in K^{\mathrm{qc}}\right\}$ is shown in figure 6 (which is bounded by the half cone $\{\operatorname{det} C \geqslant 0\}$ and one sheet of the hyperboloid $\left\{\operatorname{det} C=\delta^{2}\right\}$ ) and the half spaces

$$
\left\{C \in \mathbb{M}_{\mathrm{sym}}^{2 \times 2}: \operatorname{tr}(C(e \otimes e)) \leqslant m_{\mathcal{U}}(e)\right\}, \quad e \in S^{1} .
$$

The flat parts in the boundary of the set shown in figure 6 correspond to the two directions $e_{i}, i=1,2$, with $\left|U_{1} e_{i}\right|^{2}=\left|U_{2} e_{i}\right|^{2}=m_{\mathcal{U}}\left(e_{i}\right)$, while the intersection of the half spaces for the other normals generate the two half cones centred at $U_{1}^{\mathrm{T}} U_{1}$ and $U_{2}^{\mathrm{T}} U_{2}$. In particular, there exists no finite subset of $S^{1}$ which describes $K^{\mathrm{qc}}$, contrary to the case of two $\mathrm{SO}(2)$-invariant wells in example 3.4.

EXAmple 4.5 (The four-well problem). Assume that $a, b, c>0, a>b, a b-c^{2}>0$ and define

$$
U_{1}=\left(\begin{array}{ll}
a & c \\
c & b
\end{array}\right), \quad U_{2}=\left(\begin{array}{ll}
b & c \\
c & a
\end{array}\right), \quad U_{3}=\left(\begin{array}{cc}
a & -c \\
-c & b
\end{array}\right), \quad U_{4}=\left(\begin{array}{cc}
b & -c \\
-c & a
\end{array}\right) .
$$

Let $\mathcal{U}=\left\{U_{1}, U_{2}, U_{3}, U_{4}\right\}$ and

$$
K=\bigcup_{i=1}^{4} \mathrm{O}(2) U_{i}
$$




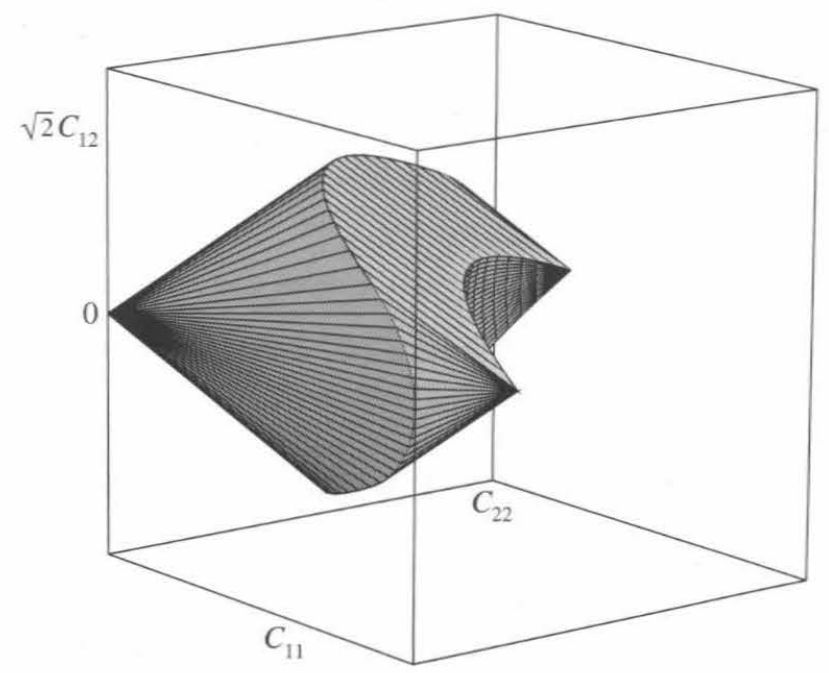

Figure 6. The set $\left(\mathrm{O}(2) U_{1} \cup \mathrm{O}(2) U_{2}\right)_{\mathrm{c}}^{\mathrm{qc}}$ for the diagonal matrices $U_{1}=\operatorname{diag}(a, b)$ and $U_{2}=\operatorname{diag}(b, a)$. This is also equal to the set $\left(\mathrm{SO}(3) \hat{U}_{1} \cup \mathrm{SO}(3) \hat{U}_{2}\right)_{\mathrm{c}}^{\mathrm{qc}}$ for the diagonal matrices $U_{1}=\operatorname{diag}(a, b, c)$ and $U_{2}=\operatorname{diag}(b, a, c)$.

Then

$$
K^{\mathrm{qc}}=\left\{F \in \mathbb{M}^{2 \times 2}: \operatorname{det} F=\delta,|F e|^{2} \leqslant m_{\mathcal{U}}(e) \forall e \in S^{1}\right\} .
$$

The set $K_{\mathrm{c}}^{\mathrm{qc}}=\left\{F^{\mathrm{T}} F: F \in K^{\mathrm{qc}}\right\}$ is shown in figure 4. The four flat parts in the boundary correspond now to the four rank-one connections shown in figure 2, which defined the boundary of the quasiconvex hull of the corresponding set with $\mathrm{SO}(2)$-invariant wells.

\section{The quasiconvex hull of $\operatorname{SO}(3) \hat{U}_{1} \cup \cdots \cup \operatorname{SO}(3) \hat{U}_{k}$}

In this section we prove theorem 1.3. We begin with an equivalent description of the set $\mathrm{SO}(3) \hat{F}$. Let $L=\left\{F \in \mathbb{M}^{3 \times 2}: F_{31}=F_{32}=0\right\}$ and define $\pi_{L}: \mathbb{M}^{2 \times 2} \rightarrow L$ by

$$
\pi_{L}(G)=\left(\begin{array}{cc}
G_{11} & G_{12} \\
G_{21} & G_{22} \\
0 & 0
\end{array}\right)
$$

Recall that $\hat{F}=\left(F e_{1}, F e_{2}\right) \in \mathbb{M}^{3 \times 2}$ for $F \in \mathbb{M}^{3 \times 3}$.

Lemma 5.1. Let $F \in \mathbb{M}^{3 \times 3}$. We have $\mathrm{SO}(3) \hat{F}=\mathrm{SO}(3) \pi_{L}(G)$, where $G$ is the square root of $\hat{F}^{\mathrm{T}} \hat{F} \in \mathbb{M}^{2 \times 2}$.

Proof. Choose a rotation $Q_{0}$ that maps the two-dimensional affine subspace spanned by the first two columns of $F$ to the subspace $\left\{s e_{1}+t e_{2}, s, t \in \mathbb{R}\right\}$. Then $Q_{0} F=\pi_{L}(G)$ for some matrix $G \in \mathbb{M}^{2 \times 2}$. Replacing $Q_{0}$ by $\left(-I+2 e_{1} \otimes e_{1}\right) Q_{0}$, or by $\left(-I+2 e_{2} \otimes e_{2}\right) Q_{0}$ if necessary, we may assume that $G$ is positive-definite. Finally, pre-multiplying $Q_{0}$ by a suitable rotation of the two-dimensional space $\left\{s e_{1}+t e_{2}, s, t \in \mathbb{R}\right\}$, we may assume that $Q_{0} F=\pi_{L}(G)$, with $G$ positive-definite 
and symmetric. By construction,

$$
(Q \hat{F})^{\mathrm{T}}(Q \hat{F})=\hat{F}^{\mathrm{T}} \hat{F}=G^{\mathrm{T}} G=G^{2},
$$

and thus $G$ is the square root of $\hat{F}^{\mathrm{T}} \hat{F}$. The assertion of the lemma now follows easily, since $\mathrm{SO}(3) \hat{F}=\mathrm{SO}(3)\left(Q_{0} \hat{F}\right)$.

Let $\hat{\mathcal{U}}=\left\{\hat{U}_{1}, \ldots, \hat{U}_{k}\right\}$, and define

$$
m_{\hat{\mathcal{U}}}(e)=\max \left\{\left|\hat{U}_{i} e\right|^{2}: i=1, \ldots, k\right\} \quad \text { for } e \in S^{1} .
$$

Proof of theorem 1.3. Let

$$
\mathcal{A}=\left\{F \in \mathbb{M}^{3 \times 2}: \operatorname{det}\left(F^{\mathrm{T}} F\right) \leqslant \delta^{2},|F e|^{2} \leqslant m_{\hat{\mathcal{U}}}(e) \forall e \in S^{1}\right\} .
$$

We first show that $K^{\mathrm{pc}} \subset \mathcal{A}$. A short calculation shows that, for all $F \in \mathbb{M}^{3 \times 2}$,

$$
\operatorname{det}\left(F^{\mathrm{T}} F\right)=\operatorname{adj}_{12}^{2}(F)+\operatorname{adj}_{13}^{2}(F)+\operatorname{adj}_{23}^{2}(F),
$$

where $\operatorname{adj}_{i j}(F)$ denotes the $(2 \times 2)$-subdeterminant formed with the $i$ th and the $j$ th rows of $F$. Thus

$$
h(F)=\left(\operatorname{det}\left(F^{\mathrm{T}} F\right)-\delta^{2}\right)_{+}
$$

is a polyconvex function on $\mathbb{M}^{3 \times 2}$. Let

$$
g_{\nu}(F)=\left(|F \nu|^{2}-m_{\hat{\mathcal{U}}}(\nu)\right)_{+} .
$$

Then

$$
\Phi(F)=h(F)+\sup _{\nu \in S^{1}} g_{\nu}(F)
$$

is a polyconvex function that is zero on $\mathcal{A}$ and positive for all $F \notin \mathcal{A}$. This proves the inclusion $K^{\mathrm{pc}} \subset \mathcal{A}$. Thus it remains to show that $\mathcal{A} \subset K^{\mathrm{lc}}$.

By lemma 5.1, we may choose $Q_{i} \in \mathrm{SO}(3)$ and $G_{i} \in \mathbb{M}_{\text {sym }}^{2 \times 2}$ positive-definite such that $Q_{i} \hat{U}_{i}=\pi_{L}\left(G_{i}\right)$. Since

$$
\operatorname{adj}_{33}\left(U_{i}^{\mathrm{T}} U_{i}\right)=\operatorname{adj}_{33}\left(\left(Q_{i} U_{i}\right)^{\mathrm{T}}\left(Q_{i} U_{i}\right)\right)=\left(\operatorname{det} G_{i}\right)^{2}=\delta^{2},
$$

we conclude that $\operatorname{det} G_{i}=\delta$ for $i=1, \ldots, k$. Moreover, if we define for $e \in S^{1}$ the vector $\tilde{e} \in \mathbb{R}^{3}$ by $\tilde{e}=\left(e_{1}, e_{2}, 0\right)$, then

$$
\left|\hat{U}_{i} e\right|^{2}=\left|U_{i} \tilde{e}\right|^{2}=\left|Q_{i} U_{i} \tilde{e}\right|^{2}=\left|G_{i} e\right|^{2},
$$

and therefore

$$
\max \left\{\left|G_{i} e\right|^{2}: i=1, \ldots, k\right\}=m_{\hat{\mathcal{U}}}(e) .
$$

Let

$$
\tilde{\mathcal{A}}=\left\{F=Q \pi_{L}(G) \in \mathbb{M}^{3 \times 2}: Q \in \mathrm{SO}(3), G \in\left(\mathrm{O}(2) G_{1} \cup \cdots \cup \mathrm{O}(2) G_{k}\right)^{\mathrm{qc}}\right\} .
$$

We claim that $\mathcal{A}=\tilde{\mathcal{A}}$. Indeed, let $F=Q \pi_{L}(G) \in \tilde{\mathcal{A}}$. Then

$$
\operatorname{det}\left(F^{\mathrm{T}} F\right)=\operatorname{det}(G)^{2} \leqslant \delta^{2} \text { and }|F e|^{2}=|G e|^{2} \leqslant m_{\hat{\mathcal{U}}}(e)
$$


by (5.1) and theorem 1.1. Thus $\tilde{\mathcal{A}} \subset \mathcal{A}$. Conversely, let $F \in \mathcal{A}$ and choose $Q \in \mathrm{SO}(3)$ such that $Q^{\mathrm{T}} F=\pi_{L}(G)$. Then

$$
\operatorname{det}\left(G^{\mathrm{T}} G\right)=\operatorname{det}\left(F^{\mathrm{T}} F\right) \leqslant \delta^{2} \text { and }|G e|^{2}=|F e|^{2} \leqslant m_{\hat{\mathcal{U}}}(e) .
$$

This proves $\mathcal{A}=\tilde{\mathcal{A}}$ and it remains to show that $\tilde{\mathcal{A}} \subset K^{\text {lc }}$. For $Q \in \mathrm{SO}(2)$, we define

$$
Q^{+}=\left(\begin{array}{ccc}
Q_{11} & Q_{12} & 0 \\
Q_{21} & Q_{22} & 0 \\
0 & 0 & 1
\end{array}\right), \quad Q^{-}=\left(-I+2 e_{2} \otimes e_{2}\right) Q^{+} \in \operatorname{SO}(3) .
$$

By the definition of $K$,

$$
Q^{ \pm} Q_{i} \hat{U}_{i} \in K
$$

and thus

$$
\pi_{L}\left(\mathrm{O}(2) G_{i}\right) \in K \quad \text { for } i=1, \ldots, n .
$$

Since $K$ is invariant under multiplication by $\mathrm{SO}(3)$ from the left, we conclude that $\mathcal{A}=\tilde{\mathcal{A}} \subset K^{\mathrm{lc}}$, and the assertion of the theorem follows.

\section{Existence of minimizers}

In this section we address the question whether there exist minimizers of the variational problem (1.1). This was an open problem for a long time, but recently fairly general positive results have been obtained in $[10,11]$ based on Baire's theorem and in $[16,17]$ based on Gromov's idea of convex integration. Following Gromov [13] and Müller and Šverák $[16,17]$, we define an in-approximation of a given set $K$ in the following way.

Definition 6.1. Let $K \subset \mathbb{M}^{m \times n}$. A sequence of open sets $V_{i} \subset \mathbb{M}^{m \times n}$ is called an in-approximation of $K$ if the following three conditions are satisfied.

(i) $V_{i} \subset V_{i+1}^{\mathrm{lc}}$.

(ii) The sets $V_{i}$ are uniformly bounded.

(iii) If a sequence $F_{i} \in V_{i}$ converges to $F \in \mathbb{M}^{m \times n}$ as $i \rightarrow \infty$, then $F \in K$.

In this definition, we replace open sets with relatively open sets, if the set $K$ is a relatively open set with respect to the constraint that one of the minors is fixed (see [17]). For example, in case (i) of theorem 1.1, the set $K$ and its generalized convex hulls are contained in the smooth manifold $\{\operatorname{det} X=\delta\}$.

We will rely on the following existence result.

THEOREM 6.2 (see $[16,17])$. Suppose that $K \subset \mathbb{M}^{m \times n}$ admits an in-approximation by (relatively) open sets $V_{i}$ in the sense of definition 6.1. Let $v \in C^{1}\left(\Omega ; \mathbb{R}^{m}\right)$ and assume that $D v(x) \in V_{1}$ for $x \in \Omega$. Then there exists a $u \in W^{1, \infty}\left(\Omega ; \mathbb{R}^{m}\right)$ such that $u=v$ on $\partial \Omega$ and $D u \in K$ almost everywhere.

In view of this result, it remains to construct in-approximations for the sets under consideration in this paper. For the case of two wells (with equal or different 
determinant), this has been done in $[16,17]$. We follow these ideas in our multi-well setting.

An important ingredient in the construction of the in-approximation is the characterization of the (relative) interior of the generalized convex hulls. Throughout this section we will assume the following hypotheses.

(H1) $\mathcal{U}=\left\{U_{1}, \ldots, U_{k}\right\}, k \geqslant 2$, and the matrices $U_{i} \in \mathbb{M}_{\text {sym }}^{2 \times 2}$ are positive-definite with $\operatorname{det} U_{i}=\delta>0$.

(H2) For all $U_{i} \in \mathcal{U}$, there exists a vector $e \in S^{1}$ such that $\left|U_{i} e\right|^{2}>m_{\mathcal{U} \backslash\left\{U_{i}\right\}}(e)$.

(See lemma 3.5 for a justification of (H2)).

Lemma 6.3. Assume (H1) and (H2) and let $K=\mathrm{SO}(2) U_{1} \cup \cdots \cup \mathrm{SO}(2) U_{k}$. Then the relative interior of $K^{\text {lc }}$ is given by

$$
\operatorname{relint}\left(K^{\mathrm{lc}}\right)=\left\{F \in K^{\mathrm{lc}}:|F e|<m_{\mathcal{U}}(e) \forall e \in S^{1}\right\} .
$$

Proof. Let $\mathcal{A}$ denote the right-hand side in (6.1) and define

$$
\mathcal{B}=\left\{F \in K^{\mathrm{lc}}: \exists e \in S^{1}:|F e|^{2}=m_{\mathcal{U}}(e)\right\} .
$$

Clearly, $K^{\text {lc }}=\mathcal{A} \cup \mathcal{B}$. If $F \in \mathcal{A}$, then there exists, by compactness of $S^{1}$, a $\delta>0$ such that $|F e|^{2} \leqslant m_{\mathcal{U}}(e)-\delta$ for all $e \in S^{1}$. By continuity of the maps $F \mapsto|F e|^{2}$, it follows that $F \in \operatorname{rel}$ int $K^{\text {lc }}$. Conversely, assume that $F \in \mathcal{B}$ with $|F e|^{2}=m_{\mathcal{U}}(e)$, $e \in S^{1}$. Let $F_{t}=F\left(I+t e^{\perp} \otimes e\right)$. Then $\operatorname{det} F_{t}=\operatorname{det} F=\delta$ and

$$
\left|F_{t} e\right|^{2}=|F e|^{2}+2 t\left\langle F e, F e^{\perp}\right\rangle+t^{2}\left|F e^{\perp}\right|^{2} .
$$

If $e$ is an eigenvector of $F^{\mathrm{T}} F$, then $F_{t} \notin K^{\mathrm{lc}}$ for all $t \neq 0$ and thus $F$ does not belong to the relative interior of $K^{\text {lc }}$. Otherwise, we conclude $F_{t} \notin K^{\text {lc }}$ for $t=s\left\langle F e, F e^{\perp}\right\rangle$ with $0<s<s_{0}$ and $s_{0}$ small enough. This proves the assertion of the lemma.

Lemma 6.4. Assume (H1) and (H2). Then there exist, for all matrices $U_{i} \in \mathcal{U}$, matrices $U_{i}^{(j)} \in \operatorname{rel} \operatorname{int}\left(K^{\mathrm{lc}}\right)$ such that $U_{i}^{(j)} \rightarrow U_{i}$ as $j \rightarrow \infty$ for $i=1, \ldots, k$. Moreover, for each compact set $E \subset \operatorname{rel} \operatorname{int}\left(K^{\mathrm{lc}}\right)$, there exists a $j_{0} \in \mathbb{N}$ such that

$$
E \subset\left(\bigcup_{i=1}^{k} \operatorname{SO}(2) U_{i}^{(j)}\right)^{\mathrm{lc}}, \quad j \geqslant j_{0} .
$$

Proof. We first construct for $U_{i} \in \mathcal{U}$ a sequence of matrices $U_{i}^{(j)} \in \operatorname{rel} \operatorname{int}\left(K^{\mathrm{lc}}\right)$ such that $U_{i}^{(j)} \rightarrow U_{i}$ as $j \rightarrow \infty$. By lemma 3.6, there exist exactly two matrices $U_{i \pm 1}$ and vectors $e_{i \pm 1} \in S^{1}, e_{i-1}$, not parallel to $e_{i+1}$, such that

$$
\left|U_{i} e_{i \pm 1}\right|^{2}=\left|U_{i \pm 1}\left(e_{i \pm 1}\right)\right|^{2}=m_{\mathcal{U}}\left(e_{i \pm 1}\right) .
$$

Thus there exist $Q_{i \pm 1} \in \mathrm{SO}(2), a_{i \pm 1} \in \mathbb{R}^{2}$ such that

$$
U_{i}-Q_{i \pm 1} U_{i \pm 1}=a_{i \pm 1} \otimes e_{i \pm 1}^{\perp} .
$$

Now let

$$
V_{i \pm 1}^{\varepsilon}=(1-\varepsilon) U_{i}+\varepsilon Q_{i \pm 1} U_{i \pm 1}
$$


By lemma 6.2 , there exists $Q_{\varepsilon} \in \mathrm{SO}(2), b \in \mathbb{R}^{2}, m \in S^{1}$ such that

$$
Q_{\varepsilon} V_{i+1}^{\varepsilon}-V_{i-1}=b \otimes m .
$$

We claim that

$$
W_{i}^{\lambda, \varepsilon}=\lambda Q_{\varepsilon} V_{i+1}^{\varepsilon}+(1-\lambda) V_{i-1}^{\varepsilon} \in \operatorname{relint} K^{\mathrm{lc}} \quad \text { for } \varepsilon \in(0,1), \quad \lambda \in(0,1) .
$$

By construction, $W_{i}^{\lambda, \varepsilon} \in K^{\mathrm{lc}}$ and therefore it suffices by lemma 6.3 to show that

$$
\left|W_{i}^{\lambda, \varepsilon} e\right|^{2}<m_{\mathcal{U}}(e) \quad \forall e \in S^{1} .
$$

This is immediate in the case $U_{i-1} \neq U_{i+1}$, since

$$
m_{\mathcal{U}}(e)=\left|W_{i}^{\lambda, \varepsilon} e\right|^{2} \leqslant(1-\varepsilon)\left|U_{i} e\right|^{2}+\lambda \varepsilon\left|U_{i+1} e\right|^{2}+(1-\lambda) \varepsilon\left|U_{i-1} e\right|^{2} \leqslant m_{\mathcal{U}}(e)
$$

implies $\left|U_{i} e\right|^{2}=\left|U_{i \pm 1} e\right|^{2}=m_{\mathcal{U}}(e)$, contradicting lemma 3.6 (i).

Assume now that $U_{i-1}=U_{i+1}$. In this case, we have, by convexity,

$$
m_{\mathcal{U}}(e)=\left|W_{i}^{\lambda, \varepsilon} e\right|^{2} \leqslant \lambda\left|V_{i+1}^{\varepsilon} e\right|^{2}+(1-\lambda)\left|V_{i-1}^{\varepsilon} e\right|^{2} \leqslant m_{\mathcal{U}}(e),
$$

and we conclude that

$$
\left|V_{i \pm 1}^{\varepsilon} e\right|^{2}=m_{\mathcal{U}}(e) .
$$

Consequently, $e=e_{i-1}$ or $e=e_{i+1}$. We may assume that the latter holds. But then, by lemma $2.2,\left|V_{i-1}^{\varepsilon} e_{i+1}\right|^{2}<m_{\mathcal{U}}\left(e_{i+1}\right)$ for $\varepsilon \in(0,1)$, and this contradicts (6.3). Thus $W_{i}^{\lambda, \varepsilon} \in \operatorname{relint}\left(K^{\mathrm{lc}}\right)$. Now define, for example, $U_{i}^{(j)}=W_{i}^{1 / j, 1 / j}$. Then $U_{i}^{(j)} \in \operatorname{rel} \operatorname{int}\left(K^{\mathrm{lc}}\right)$ and $U_{i}^{(j)} \rightarrow U_{i}$ as $j \rightarrow \infty$.

Finally, the inclusion (6.2) follows from lemma 6.3 , since, by continuity, there exists, for all $\varepsilon>0$, a $j_{0}>0$ such that

$$
\left|m_{\mathcal{U}}(e)-m_{\left\{U_{1}^{(j)}, \ldots, U_{k}^{(j)}\right\}}(e)\right|<\varepsilon \quad \forall j \geqslant j_{0} \quad \forall e \in S^{1} .
$$

After these preparations we are in a position to prove our first existence result.

TheOREM 6.5. Suppose that $W: \mathbb{M}^{2 \times 2} \rightarrow \mathbb{R}, W \geqslant 0$, that

$$
K=\left\{W^{-1}(0)\right\}=\operatorname{SO}(2) U_{1} \cup \cdots \cup \operatorname{SO}(2) U_{k}
$$

and that the matrices $U_{i}$ satisfy (H1) and (H2). Assume that $v \in C^{1}\left(\Omega ; \mathbb{R}^{m}\right)$ is such that $\{D v(x): x \in \Omega\}$ is contained in a compact subset of $\operatorname{rel} \operatorname{int}\left(K^{\mathrm{lc}}\right)$. Then there exists a minimizer $u$ of the variational problem: minimize

$$
I(w)=\int_{\Omega} W(D w) \mathrm{d} x
$$

in the class $\left\{w \in W^{1, \infty}\left(\Omega ; \mathbb{R}^{2}\right): w=v\right.$ on $\left.\partial \Omega\right\}$. In particular, $I(u)=0$.

Proof. In view of theorem 6.2 , it remains to construct an in-approximation of $K$ with relatively open sets $V_{i}$ such that $\{D v(x): x \in \Omega\} \subset V_{1}$. 
The existence of an in-approximation will be a consequence of lemmas 6.3 and 6.4. Choose $V_{1} \subset \subset$ rel int $K^{\text {lc }}$ such that $\{D v(x): x \in \Omega\} \subset \subset V_{1}$. Let $\delta_{1}>0$ be given. By lemma 6.4, we may choose $U_{i}^{(1)} \in \operatorname{rel}$ int $K^{\text {lc }}$ such that $\left|U_{i}^{(1)}-U_{i}\right|<\delta_{1}$ and

$$
\bar{V}_{1} \subset\left(\bigcup_{i=1}^{k} \operatorname{SO}(2) U_{i}^{(1)}\right)^{\mathrm{lc}} .
$$

Let $\varepsilon_{1}=\operatorname{dist}\left(\left\{\mathrm{SO}(2) U_{1}^{(j)} \cup \cdots \cup \mathrm{SO}(2) U_{k}^{(j)}\right\}, \partial \operatorname{conv}(K)\right)$ and define

$$
V_{2}=\left\{F: \operatorname{det} F=\delta, \operatorname{dist}\left(F, \operatorname{SO}(2) U_{1}^{(k)} \cup \cdots \cup \mathrm{SO}(2) U_{n}^{(k)}\right)<\frac{1}{2} \varepsilon_{1}\right\} .
$$

Then $V_{1} \subset\left(V_{2}\right)^{\text {lc }}$ and $\operatorname{dist}(F, K)<2 \delta_{1}$ for all $F \in V_{2}$. Proceeding inductively with $\delta_{1}$ replaced by $2^{-j} \delta_{1}$, we obtain an in-approximation of $K$. This proves the theorem.

We do not expect similar existence results in three dimensions when the wells are essentially two dimensional, since it is not possible to lift the two-dimensional construction in such a manner that they satisfy three-dimensional boundary condition.

REMARK 6.6. Let $K=\mathrm{SO}(3) U_{1} \cup \cdots \cup \mathrm{SO}(3) U_{k}$, where $\left\{U_{1}, \ldots, U_{k}\right\} \subset \mathbb{M}_{\text {sym }}^{3 \times 3}$ with $U_{i}$ positive-definite, $\operatorname{det} U_{i}=\delta>0$ and assume that there exists $\mu>0$ and $v \in S^{2}$ such that $U_{i} v=\mu v$ for $i=1, \ldots, k$. Assume that $\Omega$ is a unit cube with sides parallel to the orthonormal basis $\left\{e_{1}, e_{2}, v\right\}$. Then, given any $F \in K^{\mathrm{qc}} \backslash K$,

$$
I(w)=\int_{\Omega} W(D w) \mathrm{d} x
$$

has no minimizer in the class $\left\{w \in W^{1, \infty}\left(\Omega ; \mathbb{R}^{m}\right): w=F x\right.$ on $\left.\partial \Omega\right\}$.

We prove this by contradiction. Let $y$ be a minimizer. Notice that inf $I=0$, since $F \in K^{\mathrm{qc}}$. Therefore, $I(y)=0$ and consequently, $\nabla y \in K$ a.e. $x \in \Omega$, and hence

$$
(\nabla y)^{\mathrm{T}}(\nabla y) v=\mu^{2} v \quad \text { and } \quad \operatorname{det} \nabla y=\delta \quad \text { a.e. } x \in \Omega .
$$

It follows then, by theorem 3.1 of Ball and James [3], that $y$ is a plane strain deformation, i.e.

$$
y(x)=Q\left(\begin{array}{c}
y_{1}\left(x_{1}, x_{2}\right) \\
y_{2}\left(x_{1}, x_{2}\right) \\
\mu x_{3}
\end{array}\right)
$$

in an orthonormal basis parallel to $\left\{e_{1}, e_{2}, v\right\}$. Comparing with the boundary condition on the surface $x_{3}=0$, we conclude that $y=F x$ on $\Omega$. Thus

$$
I(y)=|\Omega| W(F)>0,
$$

contradicting the assumption that $y$ is a minimizer.

Now we turn to the case $K=\mathrm{O}(2) U_{1} \cup \cdots \cup \mathrm{O}(2) U_{k}$. We first prove the analogues of lemmas 6.3 and 6.4 in this situation.

Lemma 6.7. Suppose that (H1) and (H2) hold and let $K=\mathrm{O}(2) U_{1} \cup \cdots \cup \mathrm{O}(2) U_{k}$. Then the interior of $K^{\mathrm{lc}}$ is given by

$$
\operatorname{int} K^{\mathrm{lc}}=\left\{F \in K^{\mathrm{lc}}:|\operatorname{det} F|<\delta \text { and }|F e|^{2}<m_{\mathcal{U}}(e) \forall e \in S^{1}\right\} .
$$


Proof. Let $\mathcal{A}$ denote the right-hand side in (6.4) and define

$$
\mathcal{B}=\left\{F \in K^{\mathrm{lc}}:|\operatorname{det} F|=\delta \text { or } \exists e \in S^{1}:|F e|^{2}=m_{\mathcal{U}}(e)\right\} .
$$

By continuity, it is easy to see that $\mathcal{A} \subset \operatorname{int} K^{\mathrm{lc}}$. Since $K^{\mathrm{lc}}=\mathcal{A} \cup \mathcal{B}$, it suffices to show that no point in $\mathcal{B}$ is an interior point of $K^{\mathrm{lc}}$. Assume first that $|\operatorname{det} F|=\delta$. Let $F_{\varepsilon}=F\left(I+\varepsilon^{2} e \otimes e\right)$ with $e \in S^{1}$. Then $\left|F-F_{\varepsilon}\right|=\varepsilon^{2}|F e|$ and $\left|\operatorname{det} F_{\varepsilon}\right|=\left(1+\varepsilon^{2}\right)|\operatorname{det} F|$. Thus $F_{\varepsilon} \rightarrow F$ as $\varepsilon \searrow 0$, but $F_{\varepsilon} \notin K^{\text {lc }}$ for any $\varepsilon>0$. Therefore, $F$ cannot be an interior point of $K^{\mathrm{lc}}$. Assume now that $|\operatorname{det} F|<\delta$ and that there exists an $e \in S^{1}$ such that $|F e|^{2}=m_{\mathcal{U}}(e)$. It follows from lemmas 4.2 and 4.3 that there exists a $G \in B_{\delta}$ with $|F e|^{2}=|G e|^{2}$ and $\tilde{Q} \in \mathrm{SO}(2), \tilde{e} \in S^{1}$, $\lambda>0$ such that

$$
\begin{aligned}
F & =\tilde{Q}\left(G-2 \lambda \tilde{e} \otimes G^{\mathrm{T}} \tilde{e}\right) \\
& =\tilde{Q}\left(G-\frac{2 \lambda}{\left|G^{-\mathrm{T}} e^{\perp}\right|^{2}} G^{-\mathrm{T}} e^{\perp} \otimes e^{\perp}\right) .
\end{aligned}
$$

Let $F_{\varepsilon}=F+\varepsilon^{2} \tilde{Q} G e \otimes e$. Then

$$
\left|F-F_{\varepsilon}\right|=\varepsilon^{2}|G e|^{2} \quad \text { and } \quad\left|F_{\varepsilon} e\right|^{2}=\left|\tilde{Q} G e+\varepsilon^{2} \tilde{Q} G e\right|^{2}=\left(1+\varepsilon^{2}\right) m_{\mathcal{U}}(e) .
$$

Thus $F_{\varepsilon} \notin K^{\mathrm{lc}}$ for $\varepsilon \neq 0$ and hence $F$ is not an interior point of $K^{\mathrm{lc}}$. This proves the lemma.

Lemma 6.8. Assume (H1) and (H2) hold. Then there exist matrices $U_{i}^{(j)} \in \operatorname{int} K^{\mathrm{lc}}$ such that $U_{i}^{(j)} \rightarrow U_{i}$ as $j \rightarrow \infty$, for $i=1, \ldots, n$. Moreover, for each compact set $E \subset \subset \operatorname{int} K^{\mathrm{lc}}$, there exists a $k_{0} \in \mathbb{N}$ with

$$
E \subset\left(\bigcup_{i=1}^{k} \mathrm{O}(2) U_{i}^{(j)}\right)^{\mathrm{lc}}, \quad j \geqslant j_{0} .
$$

Proof. Let $\tilde{U}_{i}^{(j)}$ be the sequence of matrices constructed in lemma 6.4 , and let $U_{i}^{(j)}=\tilde{U}_{i}^{(j)}\left(I-\delta_{i, j} e \otimes e\right)$ with $e \in S^{1}$. By compactness of $S^{1}$ and continuity, we may choose $\delta_{i, j}>0$ such that $\left|U_{i}^{(j)} e\right|^{2}<m_{\mathcal{U}}(e)$ for all $e \in S^{1}$. Then

$$
\operatorname{det} U_{i}^{(j)}=\operatorname{det} \tilde{U}_{i}^{(j)}\left(1-\delta_{i, j}\right)
$$

and $U_{i}^{(j)} \rightarrow U_{i}$ if we choose, for example, $0<\delta_{i, j}<1 / j$. The inclusion (6.5) follows as in lemma 6.4 .

Theorem 6.9. Suppose that $W: \mathbb{M}^{2 \times 2} \rightarrow \mathbb{R}, W \geqslant 0$,

$$
K=\left\{W^{-1}(0)\right\}=\mathrm{O}(2) U_{1} \cup \cdots \cup \mathrm{O}(2) U_{k}
$$

and (H1) and (H2) hold. Assume that $v \in C^{1}\left(\Omega ; \mathbb{R}^{2}\right)$ is such that $\{D v(x): x \in \Omega\}$ is contained in a compact subset of int $K^{\mathrm{lc}}$. Then there exists a minimizer $u$ of the variational problem: minimize

$$
I(w)=\int_{\Omega} W(D w) \mathrm{d} x
$$

in the class $\left\{w \in W^{1, \infty}\left(\Omega ; \mathbb{R}^{2}\right): w=v\right.$ on $\left.\partial \Omega\right\}$. In particular, $I(u)=0$. 
Proof. The proof is analogous to the proof of theorem 6.5. Choose $V_{1} \subset \subset \operatorname{int} K^{\text {lc }}$ such that $\{D v(x): x \in \Omega\} \subset \subset V_{1}$. By lemma 6.8, there exist, for $\delta_{1}>0$, matrices $U_{i}^{(1)} \in \operatorname{int} K^{\text {lc }}$ such that $\left|U_{i}-U_{i}^{(1)}\right|<\delta_{1}$ and

$$
\bar{V}_{1} \subset\left(\bigcup_{i=1}^{k} \mathrm{O}(2) U_{i}^{(1)}\right)^{\mathrm{lc}} \text {. }
$$

Let $\varepsilon_{1}=\operatorname{dist}\left(\mathrm{O}(2) U_{1}^{(1)} \cup \cdots \cup \mathrm{O}(2) U_{k}^{(1)}, \partial K^{\mathrm{lc}}\right)$, and define

$$
V_{2}=\left\{F: \operatorname{dist}\left(F, \mathrm{O}(2) U_{1}^{(1)} \cup \cdots \cup \mathrm{O}(2) U_{n}^{(1)}\right)<\frac{1}{2} \varepsilon\right\} .
$$

Then $V_{1} \subset\left(V_{2}\right)^{\text {lc }}$ and $\operatorname{dist}(F, K)<2 \delta_{1}$ for all $F \in V_{2}$. Proceeding iteratively, we obtain the required in-approximation. This proves the theorem.

Finally, we prove an existence result for the $\mathrm{SO}(3)$-invariant wells. This requires first a modification of (H1) and (H2). We will assume the following.

$\left(\mathrm{H} 1^{\prime}\right) \mathcal{U}=\left\{U_{1}, \ldots, U_{k}\right\}, k \geqslant 2$, and the matrices $U_{i} \in \mathbb{M}_{\text {sym }}^{3 \times 3}$ are positive-definite with $\operatorname{adj}_{33}\left(U_{i}^{\mathrm{T}} U_{i}\right)=\delta^{2}>0$.

$\left(\mathrm{H} 2^{\prime}\right)$ Let $Q_{i} \in \mathrm{SO}(3)$ and $G_{i} \in \mathbb{M}^{2 \times 2}$ be the matrices constructed in lemma 5.1 with $\pi_{L}\left(G_{i}\right)=Q_{i} \hat{U}_{i}$ and let $\mathcal{G}=\left\{G_{1}, \ldots, G_{k}\right\}$. Then there exists, for all $G_{i} \in \mathcal{G}$, an $e \in S^{1}$ such that $\left|G_{i} e\right|^{2}>m_{\mathcal{G} \backslash\left\{G_{i}\right\}}(e)$.

Lemma 6.10. Assume that $\left(H 1^{\prime}\right)$ and $\left(H 2^{\prime}\right)$ hold. Let $K=\operatorname{SO}(3) \hat{U}_{1} \cup \cdots \cup \operatorname{SO}(3) \hat{U}_{k}$. Then

$$
\text { int } K^{\mathrm{lc}}=\left\{F \in \mathbb{M}^{3 \times 2}: \operatorname{det}\left(F^{\mathrm{T}} F\right)<\delta^{2},|F e|^{2}<m_{\hat{\mathcal{U}}}(e) \forall e \in S^{1}\right\} .
$$

Proof. Let $\mathcal{A}$ denote the right-hand side in (6.6) and define

$$
\mathcal{B}=\left\{F \in K^{\mathrm{lc}}: \operatorname{det}\left(F^{\mathrm{T}} F\right)=\delta^{2} \text { or } \exists e \in S^{1}:|F e|^{2}=m_{\hat{\mathcal{U}}}(e)\right\} .
$$

Then $K^{\text {lc }}=\mathcal{A} \cup \mathcal{B}$, and by continuity it is easy to see that $\mathcal{A} \subset \operatorname{int} K^{\text {lc }}$. Conversely, assume that $F \in \mathcal{B}$. Since $F$ is not an interior point of $K^{\mathrm{lc}}$ if and only if $Q F$ is not an interior point for some $Q \in \mathrm{SO}(3)$, we may assume that $F=\pi_{L}(G)$ with $G \in\left(\mathrm{O}(2) G_{1} \cup \cdots \cup \mathrm{O}(2) G_{n}\right)^{1 \mathrm{c}}$ and $\operatorname{det}\left(G^{\mathrm{T}} G\right)=\delta^{2}$ or $|G e|^{2}=m_{\hat{\mathcal{u}}}(e)$ (see the proof of lemma 5.1). We conclude, as in the proof of lemma 6.7 , that $G$ is not an interior point of $\left(\mathrm{O}(2) G_{1} \cup \cdots \cup \mathrm{O}(2) G_{k}\right)^{\mathrm{lc}}$, and this implies the assertion of the lemma.

Lemma 6.11. Assume that $\left(H 1^{\prime}\right)$ and $\left(H 2^{\prime}\right)$ hold. Let $K=\operatorname{SO}(3) \hat{U}_{1} \cup \cdots \cup \operatorname{SO}(3) \hat{U}_{k}$. Then there exist positive-definite matrices $U_{i}^{(j)} \in \mathbb{M}^{3 \times 3}$ such that $\hat{U}_{i}^{(j)} \in \operatorname{int} K^{\mathrm{lc}}$ and $U_{i}^{(j)} \rightarrow U_{i}$ as $j \rightarrow \infty$. Moreover, for each compact set $E \subset \subset \operatorname{int} K^{\mathrm{lc}}$, there exists a $j_{0} \in \mathbb{N}$ with

$$
E \subset\left(\bigcup_{i=1}^{k} \operatorname{SO}(3) \hat{U}_{i}^{(j)}\right)^{\mathrm{lc}}, \quad j \geqslant j_{0} .
$$

Proof. We may assume that $\hat{U}_{i}=\pi_{L}\left(G_{i}\right)$, with $G_{i}$ as in lemma 5.1. Let $G_{i}^{(j)}$ be the sequence of matrices constructed in lemma 6.8. Then $\pi_{L}\left(G_{i}\right) \in \operatorname{int} K^{\mathrm{lc}}$, and, by 
lemma 6.10 , the matrices

$$
U_{i}^{(j)}=\left(\begin{array}{ccc}
G_{i, 11}^{(k)} & G_{i, 12}^{(k)} & U_{i, 13} \\
G_{i, 21}^{(k)} & G_{i, 22}^{(k)} & U_{i, 23} \\
0 & & U_{i, 33}
\end{array}\right)
$$

have the properties stated in the lemma if we choose $j$ big enough, since the set of positive-definite matrices is open.

Theorem 6.12. Suppose that $W: \mathbb{M}^{3 \times 2} \rightarrow \mathbb{R}, W \geqslant 0$,

$$
K=\left\{W^{-1}(0)\right\}=\mathrm{SO}(3) \hat{U}_{1} \cup \cdots \cup \mathrm{SO}(3) \hat{U}_{n}
$$

and $\left(H 1^{\prime}\right)$ and $\left(H 2^{\prime}\right)$ hold. Assume that $v \in C^{1}\left(\Omega ; \mathbb{R}^{3}\right)$ is such that $\{D v(x): x \in \Omega\}$ is contained in a compact subset of int $K^{\mathrm{lc}}$. Then there exists a minimizer $u$ of the variational problem: minimize

$$
I(w)=\int_{\Omega} W(D w) \mathrm{d} x
$$

in the class $\left\{w \in W^{1, \infty}\left(\Omega ; \mathbb{R}^{3}\right): w=v\right.$ on $\left.\partial \Omega\right\}$. In particular, $I(u)=0$.

Proof. This is analogous to the proof of theorem 6.9.

\section{Uniqueness and non-uniqueness of microstructures}

As discussed in $\S 1$, the direct method in the calculus of variations based on weak lower semicontinuity cannot be applied to obtain existence for the variational problem (1.1). Minimizing sequences typically develop finer and finer oscillations (microstructures) and converge only weakly but not strongly. However, under suitable coercivity and growth assumptions on $W$, subsequences of the deformation gradients $\left\{D u_{k}\right\}$ of minimizing sequences generate a gradient Young measure which captures the essential statistics of the oscillations in $\left\{D u_{k}\right\}$ (see $[1,14,20]$ ). It is a natural question to ask whether the oscillations in the minimizing sequences are unique in the sense that the generated gradient Young measures are unique. In this section we prove that this is only true for some exceptional cases where the measure $\mu$ is of the form $\mu=\lambda \delta_{A}+(1-\lambda) \delta_{B}$ for $A, B \in K$ with $\operatorname{rank}(A-B)=1$. For $F \in K^{\mathrm{qc}}$, we define

$$
\mathcal{M}(F)=\{\mu: \mu \text { is gradient Young measure with } \operatorname{supp} \mu \subset K,\langle\mu, \text { id }\rangle=F\} .
$$

In order to prove our non-uniqueness results, we will use a special subset of all gradient Young measures, the so-called laminates (see, for example, [18]). Assume that $F=\lambda A+(1-\lambda) B$ with $\operatorname{rank}(A-B)=1$ and $\lambda \in(0,1)$. Then $\mu=\lambda \delta_{A}+$ $(1-\lambda) \delta_{B} \in \mathcal{M}(F)$. This process of splitting matrices in convex combinations along rank-one lines can be iterated. If $B=\mu C+(1-\mu) D$ with $\operatorname{rank}(C-D)=1$ and $\mu \in(0,1)$, then $\mu=\lambda \delta_{A}+(1-\lambda)\left(\mu \delta_{C}+(1-\mu) \delta_{D}\right) \in \mathcal{M}(F)$. In particular, we will use the following result, which follows from [4].

Proposition 7.1. Assume that $U_{1}$ and $U_{2}$ are symmetric and positive-definite with $\operatorname{det} U_{1}=\operatorname{det} U_{2}=\delta>0$. If $F \in\left(\mathrm{SO}(2) U_{1} \cup \mathrm{SO}(2) U_{2}\right)^{\mathrm{qc}}$ satisfies $|F e|^{2}<$ 
$m_{\left\{U_{1}, U_{2}\right\}}(e)$ for all $e \in S^{1}$, then $\mathcal{M}(F)$ contains at least two laminates supported on three matrices.

We first consider the case of $\mathrm{SO}(2)$-invariant wells. Let $\mathcal{U}=\left\{U_{1}, \ldots, U_{k}\right\}$ and assume that the hypotheses $(\mathrm{H} 1)$ and $(\mathrm{H} 2)$ defined in $\S 6$ hold. Recall the set $\mathcal{B}$ defined in (3.2). The following proposition shows that the Young measure is unique if and only if $F^{\mathrm{T}} F$ lies on the boundary of $K_{\mathrm{c}}^{\mathrm{qc}}$ relative to the hyperboloid $\operatorname{det} C=\delta^{2}$.

Proposition 7.2. Let $K=\mathrm{SO}(2) U_{1} \cup \cdots \cup \mathrm{SO}(2) U_{k}$ and $F \in K^{\mathrm{qc}}$.

(i) If $F \in \mathcal{B}$, i.e. if there exist an $e \in S^{1}$ and $U_{i}, U_{j} \in \mathcal{U}, i \neq j$, such that $|F e|^{2}=\left|U_{i} e\right|^{2}=\left|U_{j} e\right|^{2}>m_{\mathcal{U} \backslash\left\{U_{i}, U_{j}\right\}}(e)$, then $\mathcal{M}(F)$ contains a unique element. Indeed, there exist unique $Q_{i}, Q_{j} \in \mathrm{SO}(2)$ and $\lambda \in[0,1]$ such that

$$
\mathcal{M}(F)=\left\{\lambda \delta_{Q_{i} U_{i}}+(1-\lambda) \delta_{Q_{j} U_{j}}\right\} .
$$

(ii) If $F \notin \mathcal{B}$, i.e. if $|F e|<m_{\mathcal{U}}(e)$ for all $e \in S^{1}$, then $\mathcal{M}(F)$ contains more than one element.

Proof. Assume that $\mu \in \mathcal{M}(F)$ and let $\mu=\lambda_{1} \mu_{1}+\cdots+\lambda_{k} \mu_{k}$, where $\mu_{i}$ is a probability measure supported on $\mathrm{SO}(2) U_{i}$ and $\lambda_{i} \in[0,1]$ with $\lambda_{1}+\cdots+\lambda_{k}=1$. By Jensen's inequality,

$$
|F e|^{2} \leqslant \sum_{j=1}^{k} \lambda_{j} \int_{\operatorname{supp} \mu_{j}}|A e|^{2} \mathrm{~d} \mu_{j}(e) .
$$

The assumptions in (i) imply that $\lambda_{\ell}=0$ for $\ell \notin\{i, j\}$ and thus $\operatorname{supp} \mu \subset \mathrm{SO}(2) U_{i} \cup$ $\mathrm{SO}(2) U_{j}$. Moreover,

$$
\int_{\operatorname{supp} \mu}|A e|^{2} \mathrm{~d} \mu(A)-|F e|^{2}=\int_{\operatorname{supp} \mu}|(F-A) e|^{2} \mathrm{~d} \mu(A)=0,
$$

and therefore $\mu=\lambda \delta_{Q_{i} U_{i}}+(1-\lambda) \delta_{Q_{j} U_{j}}$, where $Q_{i}$ and $Q_{j}$ are the uniquely defined rotations with $Q_{i} U_{i} e=Q_{j} U_{j} e=F e$. Since $U_{i} \neq U_{j}$, it follows that $\lambda$ is uniquely defined and this implies (i).

To prove (ii), we consider $F(t, v)=F\left(I+t v \otimes v^{\perp}\right)$. Then $\operatorname{det} F(t, v)=\operatorname{det} F$ and there exist $t^{+}>0>t^{-}$such that $F\left(t^{ \pm}, v\right) \in \mathcal{B}$. We may assume that $F\left(t^{+}, v\right) \notin K$. By lemma 3.3 , there exist $U_{i}, U_{j} \in \mathcal{U}$ such that $F\left(t^{+}, v\right) \in\left(\operatorname{SO}(2) U_{i} \cup \operatorname{SO}(2) U_{j}\right)^{\mathrm{qc}}$. It follows from example 3.4 that $F(t, v) \in\left(\operatorname{SO}(2) U_{i} \cup \operatorname{SO}(2) U_{j}\right)^{\mathrm{qc}}$ for $t \in\left(t^{+}-\varepsilon, t^{+}\right)$, $\varepsilon$ small enough, with $\left|F\left(t^{+}, v\right)\right|^{2}<m_{\left\{U_{i}, U_{j}\right\}}(e)$ for all $e \in S^{1}$. Let $F_{0}=F\left(t_{0}, v\right)$ with $t_{0} \in\left(t^{+}-\varepsilon, t^{+}\right)$. Then there exists $\lambda \in[0,1]$ such that $F=\lambda F_{0}+(1-\lambda) F\left(t^{-}, v\right)$ and $F_{0}-F\left(t^{-}, v\right)=\alpha v \otimes v^{\perp}, \alpha \in \mathbb{R}$. The assertion follows now from proposition 7.1, since $\mathcal{M}\left(F_{0}\right)$ contains at least two laminates.

ExAmple 7.3 (The four-well problem). Consider the four-well problem described in example 3.7 . Let

$$
e_{1}=\frac{1}{\sqrt{ } 2}\left(\begin{array}{l}
1 \\
1
\end{array}\right), \quad e_{2}=\frac{1}{\sqrt{ } 2}\left(\begin{array}{c}
1 \\
-1
\end{array}\right), \quad e_{3}=\left(\begin{array}{l}
1 \\
0
\end{array}\right), \quad e_{4}=\left(\begin{array}{l}
0 \\
1
\end{array}\right)
$$


Then $\mathcal{M}(F)$ contains a unique element if and only if $F=Q U_{1}\left(I+\alpha e_{1} \otimes e_{1}^{\perp}\right)$ or $F=Q U_{2}\left(I+\alpha e_{4} \otimes e_{4}^{\frac{1}{4}}\right)$ or $F=Q U_{4}\left(I+\alpha e_{2} \otimes e_{2}^{\perp}\right)$ or $F=Q U_{3}\left(I+\alpha e_{3} \otimes e_{3}^{\perp}\right)$ for some $Q \in \mathrm{SO}(2)$ and for some $\alpha \in \mathbb{R}$. These correspond to the boundary arcs shown in dark in figure 2. In particular, note that $F$ corresponding to some rank-one laminates have more than one element in $\mathcal{M}(F)$, as, for example, $F=Q U_{1}\left(I+\alpha e_{2} \otimes e_{2}^{\frac{1}{2}}\right)$ or $F=Q U_{2}\left(I+\alpha e_{3} \otimes e_{3}^{\frac{1}{2}}\right)$ or $F=Q U_{4}\left(I+\alpha e_{1} \otimes e_{1}^{\perp}\right)$ or $F=Q U_{3}\left(I+\alpha e_{4} \otimes e_{4}^{\frac{1}{4}}\right)$, which correspond to the dashed arcs shown in figure 2 .

Very similar results hold in three dimensions when the wells are essentially two dimensional. Let $U_{i}$ satisfy the conditions of theorem 1.2.

Proposition 7.4. Let $K=\mathrm{SO}(3) U_{1} \cup \cdots \cup \mathrm{SO}(3) U_{k}$ and $F \in K^{\mathrm{qc}}$.

(i) If $F \in \mathcal{B}$, i.e. if there exist an $e \in S^{2}$ satisfying $\langle e, v\rangle=0$ and $U_{i}, U_{j} \in \mathcal{U}$, $i \neq j$, such that $|F e|^{2}=\left|U_{i} e\right|^{2}=\left|U_{j} e\right|^{2}>m_{\mathcal{U} \backslash\left\{U_{i}, U_{j}\right\}}(e)$, then $\mathcal{M}(F)$ contains a unique element. Indeed, there exist unique $Q_{i}, Q_{j} \in \mathrm{SO}(3)$ and $\lambda \in[0,1]$ such that

$$
\mathcal{M}(F)=\left\{\lambda \delta_{Q_{i} U_{i}}+(1-\lambda) \delta_{Q_{j} U_{j}}\right\} .
$$

(ii) If $F \notin \mathcal{B}$, i.e. if $|F e|<m_{\mathcal{U}}(e)$ for all $e \in S^{2}$ satisfying $\langle e, v\rangle=0$, then $\mathcal{M}(F)$ contains more than one element.

Proof. The proof follows that of proposition 7.2 , aided by the observation that $F \in K^{\mathrm{qc}}$ satisfies $\langle F e, F v\rangle=0$ for all $e \in S^{2}$ such that $\langle e, v\rangle=0$.

We now turn to the $\mathrm{O}(2)$-invariant wells. Let $\mathcal{U}=\left\{U_{1}, \ldots, U_{k}\right\}$ and assume that the hypotheses (H1) and (H2) defined in $\S 6$ hold. Recall the set $\mathcal{B}$ defined in (4.2). The following proposition shows that the Young measure is unique if and only if $F^{\mathrm{T}} F$ lies on either the cones with apex $U_{i}^{2}$ or on the intersection of the flat boundary parts with the hyperboloid $\operatorname{det} C=\delta^{2}$ in $K_{\mathrm{c}}^{\mathrm{qc}}$.

Proposition 7.5. Let $K=\mathrm{O}(2) U_{1} \cup \cdots \cup \mathrm{O}(2) U_{k}$ and $F \in K^{\mathrm{qc}}$.

(i) If there exists an $e \in S^{1}$ such that $|F e|^{2}=\left|U_{i} e\right|^{2}>m_{\mathcal{U} \backslash\left\{U_{i}\right\}}$, then $\mathcal{M}(F)$ contains a unique element. Indeed, there exist unique $\lambda \in[0,1]$ and $Q^{ \pm} \in \mathrm{O}(2)$ with $\operatorname{det} Q^{ \pm}= \pm 1$ such that

$$
\mathcal{M}(F)=\left\{\lambda \delta_{Q^{+} U_{i}}+(1-\lambda) \delta_{Q^{-} U_{i}}\right\} .
$$

(ii) If there exists an $e \in S^{1}$ such that $|F e|^{2}=\left|U_{i} e\right|^{2}=\left|U_{j} e\right|^{2}>m_{\mathcal{U} \backslash\left\{U_{i}, U_{j}\right\}}(e)$, $i \neq j$, then there exist unique $Q_{i}^{ \pm}, Q_{j}^{ \pm} \in \mathrm{O}(2)$ satisfying

$$
Q_{i}^{ \pm} U_{i} e=Q_{j}^{ \pm} U_{j} e=F e, \quad \operatorname{det} Q_{i}^{ \pm}= \pm 1, \quad \operatorname{det} Q_{j}^{ \pm}= \pm 1
$$

such that

$$
\begin{aligned}
\mathcal{M}(F)=\{\mu & \lambda_{i}^{+} \delta_{Q_{i}^{+} U_{i}}+\lambda_{i}^{-} \delta_{Q_{i}^{-} U_{i}}+\lambda_{j}^{+} \delta_{Q_{j}^{+} U_{j}}+\lambda_{j}^{-} \delta_{Q_{j}^{-} U_{j}}, \\
& \lambda_{i}^{ \pm}, \lambda_{j}^{ \pm} \in[0,1], \lambda_{i}^{+}+\lambda_{i}^{-}+\lambda_{j}^{+}+\lambda_{j}^{-}=1, \\
& \left(\lambda_{i}^{+}+\lambda_{j}^{+}\right) \delta-\left(\lambda_{i}^{-}+\lambda_{j}^{-}\right) \delta=\operatorname{det} F, \\
& \left.\left(\lambda_{i}^{+}+\lambda_{i}^{-}\right)\left\langle U_{i} e, U_{i} e^{\perp}\right\rangle+\left(\lambda_{j}^{+}+\lambda_{j}^{-}\right)\left\langle U_{j} e, U_{j} e^{\perp}\right\rangle=\left\langle F e, F e^{\perp}\right\rangle\right\} .
\end{aligned}
$$


Therefore, the set $\mathcal{M}(F)$ contains a unique element if $\operatorname{det} F= \pm \delta$ or if $\left\langle U_{i} e, U_{i} e^{\perp}\right\rangle=\left\langle F e, F e^{\perp}\right\rangle$ or if $\left\langle U_{j} e, U_{j} e^{\perp}\right\rangle=\left\langle F e, F e^{\perp}\right\rangle$. Otherwise, $\mathcal{M}(F)$ consists of a one-parameter family of measures.

(iii) If $|F e|^{2}<m_{\mathcal{U}}(e)$ for all $e \in S^{1}$, then $\mathcal{M}(F)$ contains more than one element. Proof. (i) It follows as in the proof of proposition 7.2 (i) that $\operatorname{supp} \mu \subset \mathrm{O}(2) U_{i}$ and that

$$
\int_{\operatorname{supp} \mu}|(F-A) e|^{2} \mathrm{~d} \mu(A)=0 .
$$

Since there are exactly two elements $Q^{ \pm} \in \mathrm{O}(2)$ that satisfy $Q^{ \pm} U_{i} e=F e$ (one rotation and one reflection), the assertion follows.

(ii) We note that there exist exactly four elements $Q_{i}^{ \pm}, Q_{j}^{ \pm} \in \mathrm{O}(2)$ that satisfy

$$
Q_{i}^{ \pm} U_{i} e=Q_{j}^{ \pm} U_{j} e=F e .
$$

Set

$$
\begin{aligned}
\tilde{\mathcal{M}}=\{\mu & =\lambda_{i}^{+} \delta_{Q_{i}^{+} U_{i}}+\lambda_{i}^{-} \delta_{Q_{i}^{-} U_{i}}+\lambda_{j}^{+} \delta_{Q_{j}^{+} U_{j}}+\lambda_{j}^{-} \delta_{Q_{j}^{-} U_{j}}, \\
& \lambda_{i}^{ \pm}, \lambda_{j}^{ \pm} \in[0,1], \lambda_{i}^{+}+\lambda_{i}^{-}+\lambda_{j}^{+}+\lambda_{j}^{-}=1, \\
& \left(\lambda_{i}^{+}+\lambda_{j}^{+}\right) \delta-\left(\lambda_{i}^{-}+\lambda_{j}^{-}\right) \delta=\operatorname{det} F, \\
& \left.\left(\lambda_{i}^{+}+\lambda_{i}^{-}\right)\left\langle U_{i} e, U_{i} e^{\perp}\right\rangle+\left(\lambda_{j}^{+}+\lambda_{j}^{-}\right)\left\langle U_{j} e, U_{j} e^{\perp}\right\rangle=\left\langle F e, F e^{\perp}\right\rangle\right\} .
\end{aligned}
$$

Now assume that $\mu \in \mathcal{M}(F)$. Then it follows as in the proof of proposition 7.2 (i) that

$$
\begin{gathered}
\mu=\lambda_{i}^{+} \delta_{Q_{i}^{+} U_{i}}+\lambda_{i}^{-} \delta_{Q_{i}^{-} U_{i}}+\lambda_{j}^{+} \delta_{Q_{j}^{+} U_{j}}+\lambda_{j}^{-} \delta_{Q_{j}^{-} U_{j}}, \\
\lambda_{i}^{ \pm}, \lambda_{j}^{ \pm} \in[0,1], \quad \lambda_{i}^{+}+\lambda_{i}^{-}+\lambda_{j}^{+}+\lambda_{j}^{-}=1 .
\end{gathered}
$$

Further, the requirement $\langle\mu, \mathrm{id}\rangle=F$ implies that

$$
\left(\lambda_{i}^{+} Q_{i}^{+} U_{i}+\lambda_{i}^{-} Q_{i}^{-} U_{i}+\lambda_{j}^{+} Q_{j}^{+} U_{j}+\lambda_{j}^{-} Q_{j}^{-} U_{j}\right) e^{\perp}=F e^{\perp} .
$$

Note that

$$
Q_{\alpha}^{+}=\left(I-2 \frac{Q_{\alpha}^{-} U_{\alpha}^{-\mathrm{T}} e^{\perp} \otimes Q_{\alpha}^{-} U_{\alpha}^{-\mathrm{T}} e^{\perp}}{\left|U_{\alpha}^{-\mathrm{T}} e^{\perp}\right|^{2}}\right) Q_{\alpha}^{-}, \quad \alpha=i, j,
$$

and hence we conclude that

$$
\begin{aligned}
\left(\lambda_{i}^{+}+\lambda_{i}^{-}\right) Q_{i}^{-} U_{i} e^{\perp}+ & \left(\lambda_{j}^{+}+\lambda_{j}^{-}\right) Q_{j}^{-} U_{j} e^{\perp} \\
& -\frac{2 \lambda_{i}^{+}}{\left|U_{i}^{-\mathrm{T}} e^{\perp}\right|^{2}} Q_{i}^{-} U_{i} e^{\perp}-\frac{2 \lambda_{j}^{+}}{\left|U_{j}^{-\mathrm{T}} e^{\perp}\right|^{2}} Q_{j}^{-} U_{j} e^{\perp}=F e^{\perp} .
\end{aligned}
$$

We take the inner product of this equation with $F e$, recall (7.1) and obtain

$$
\left(\lambda_{i}^{+}+\lambda_{i}^{-}\right)\left\langle U_{i} e, U_{i} e^{\perp}\right\rangle+\left(\lambda_{j}^{+}+\lambda_{j}^{-}\right)\left\langle U_{j} e, U_{j} e^{\perp}\right\rangle=\left\langle F e, F e^{\perp}\right\rangle .
$$

We obtain the final condition,

$$
\left(\lambda_{i}^{+}+\lambda_{j}^{+}\right) \delta-\left(\lambda_{i}^{-}+\lambda_{j}^{-}\right) \delta=\operatorname{det} F,
$$


by taking the cross product $\left(a \wedge b=a_{1} b_{2}-a_{2} b_{1}\right.$ for $\left.a, b \in \mathbb{R}^{2}\right)$ of $(7.2)$ with $F e$, recalling (7.1) and noting that for any $A \in \mathbb{M}^{2 \times 2}$, det $A=(A e) \wedge\left(A e^{\perp}\right)$. We have proved that $\mu \in \tilde{\mathcal{M}}$ or $\mathcal{M}(F) \subset \tilde{\mathcal{M}}$.

To prove the converse inclusions, let $\mu \in \mathcal{M}$. We note that (7.1) implies that there exist $a, b, c \in \mathbb{R}^{2}$ such that

$$
\begin{aligned}
Q_{i}^{+} U_{i}-Q_{i}^{-} & U_{i}=a \otimes e^{\perp}, \quad Q_{j}^{+} U_{j}-Q_{j}^{-} U_{j}=b \otimes e^{\perp}, \\
\left(\frac{\lambda_{i}^{+}}{\lambda_{i}^{+}+\lambda_{i}^{-}} Q_{i}^{+} U_{i}\right. & \left.+\frac{\lambda_{i}^{-}}{\lambda_{i}^{+}+\lambda_{i}^{-}} Q_{i}^{-} U_{i}\right) \\
& -\left(\frac{\lambda_{j}^{+}}{\lambda_{j}^{+}+\lambda_{j}^{-}} Q_{j}^{+} U_{j}+\frac{\lambda_{j}^{-}}{\lambda_{j}^{+}+\lambda_{j}^{-}} Q_{j}^{-} U_{j}\right)=c \otimes e^{\perp} .
\end{aligned}
$$

This implies that $\mu$ is a gradient Young measure (in fact, as a laminate of rank two). It remains to be shown that $\langle\mu, \mathrm{id}\rangle=F$. In view of (7.1), we only have to show (7.2). However, this readily follows from the last two conditions in the definition of $\mathcal{M}$ and the calculations above, since, for any $u \neq 0, v, w \in \mathbb{R}^{2}$,

$$
\langle u, v\rangle=\langle u, w\rangle \text { and } u \wedge v=u \wedge w \quad \Leftrightarrow \quad v=w .
$$

(iii) The construction in the proof of proposition 7.2 implies non-uniqueness for the case $\operatorname{det} F=\delta$ and also for $\operatorname{det} F=-\delta$ (by pre-multiplying every matrix in the construction by $J=\operatorname{diag}(-1,1))$. Consider next the case $\operatorname{det} F=0$. We may assume that $F=\alpha v \otimes v$ with $\alpha>0$. Since, by assumption, $|F e|^{2}<m_{\mathcal{U}}(e)$ for all $e \in S^{1}$, there exists a $\bar{\alpha}>\alpha$ such that $\bar{\alpha} v \otimes v \in \mathcal{B}$. Let $\lambda=\alpha / \bar{\alpha}$. Then $F=(1-\lambda) 0+\lambda \bar{F}$, and since there is more than one laminate with centre of mass equal to zero, the assertion follows. Consider finally the case $0<|\operatorname{det} F|<\delta$. We may assume that $0<\operatorname{det} F=\gamma<\delta$. Choose any $G \in \mathcal{B}_{\delta}$ of the form $G=\lambda Q U_{i}+(1-\lambda) U_{j}$ with $\lambda \in(0,1), Q \in \mathrm{SO}(2)$ and $Q U_{i}-U_{j}=a \otimes e^{\perp}$. Let $G_{t}=G-2 t \tilde{e} \otimes G^{\mathrm{T}} \tilde{e}$ with $\tilde{e}=G^{-\mathrm{T}} e^{\perp} /\left|G^{-\mathrm{T}} e^{\perp}\right|$. Since $\operatorname{det} G_{t}=\operatorname{det} G(1-2 t)$, there exists a $\bar{t} \in\left(0, \frac{1}{2}\right)$ such that $\bar{G}=G_{\bar{t}}$ satisfies $\operatorname{det} \bar{G}=\gamma$. By lemma 2.2 , there exists $R \in \operatorname{SO}(2), a, b \in \mathbb{R}^{2}$ such that $F=R \bar{G}+a \otimes b$. Let $F_{\alpha}=R \bar{G}+\alpha a \otimes b$; clearly, there exist $\alpha_{0}>1$ such that $F_{\alpha_{0}} \in \mathcal{B}_{\gamma}$ and $F_{\alpha_{0}} \neq R \bar{G}$. Therefore, we can obtain $F$ by laminating $\bar{G}$ and $F_{\alpha_{0}}$; the result follows since the proof of (ii) shows that $\mathcal{M}(\bar{G})$ contains more than one laminate.

We finally turn to the case of the thin-film wells. Let $\mathcal{U}=\left\{U_{1}, \ldots, U_{k}\right\}$ and assume that the hypotheses $\left(\mathrm{H}^{\prime}\right)$ and $\left(\mathrm{H}^{\prime}\right)$ defined in $\S 6$ hold. The result says that the Young measure is unique if and only if $F^{\mathrm{T}} F$ lies on the intersection of the flat boundary regions with the hyperboloid $\operatorname{det} C=\delta^{2}$ in $K_{\mathrm{c}}^{\mathrm{qc}}$. Notice that, unlike the case of the $\mathrm{O}(2)$-invariant wells, there is no uniqueness in the cones, since we can make new constructions which use the third dimension.

Proposition 7.6. Let $K=\mathrm{SO}(3) \hat{U}_{1} \cup \cdots \cup \mathrm{SO}(3) \hat{U}_{k}$ and $F \in K^{\mathrm{qc}}$. Then $\mathcal{M}(F)$ contains a unique element if and only if $\operatorname{det}\left(F^{\mathrm{T}} F\right)=\delta^{2}$ and there exists an $e \in S^{1}$ such that

$$
|F e|^{2}=\left|\hat{U}_{i} e\right|^{2}=\left|\hat{U}_{j} e\right|^{2}>m_{\hat{\mathcal{U}} \backslash\left\{\hat{U}_{i}, \hat{U}_{j}\right\}}(e), \quad i \neq j .
$$


Proof. Consider first the case $\operatorname{det}\left(F^{\mathrm{T}} F\right)=\delta^{2}$ and assume that there exists an $e \in S^{1}$ such that $|F e|^{2}=\left|\hat{U}_{i} e\right|^{2}=\left|\hat{U}_{j} e\right|^{2}>m_{\hat{\mathcal{U}} \backslash\left\{\hat{U}_{i}, \hat{U}_{j}\right\}}(e), i \neq j$. We can adopt the proof of proposition 7.2 to establish that the Young measure is unique.

Now consider $F$ such that there exists an $e \in S^{1}$ such that

$$
|F e|^{2}=\left|\hat{U}_{i} e\right|^{2}>m_{\hat{\mathcal{U}} \backslash\left\{\hat{U}_{i}\right\}} .
$$

We show that $\mathcal{M}(F)$ contains more than one element. We may assume that $F e=$ $\hat{U}_{i} e$ or $F=(I-2 \lambda v \otimes v) \hat{U}_{i}$, where $v \cdot \hat{\mathcal{U}}_{i} e=v \cdot w=0$ for $w=\{0,0,1\}$. In an orthonormal basis $\{u, v, w\}$,

$$
\hat{U}_{i}=\left(\begin{array}{ll}
a & b \\
c & d \\
0 & 0
\end{array}\right) \quad \text { and } \quad F=\left(\begin{array}{cc}
a & b \\
(1-2 \lambda) c & (1-2 \lambda) d \\
0 & 0
\end{array}\right) .
$$

Let

$$
R_{\theta}^{ \pm}=\left(\begin{array}{ccc}
1 & 0 & 0 \\
0 & \cos \theta & \mp \sin \theta \\
0 & \pm \sin \theta & \cos \theta
\end{array}\right)
$$

Note that $R_{\theta}^{ \pm} \in \mathrm{SO}(3)$ and that

$$
R_{\theta}^{+} \hat{U}_{i}-R_{\theta}^{-} \hat{U}_{i}=\left(R_{\theta}^{+}-R_{\theta}^{-}\right) \hat{U}_{i}=2 \sin \theta\left(\begin{array}{ll}
0 & 0 \\
0 & 0 \\
c & d
\end{array}\right)
$$

is rank-one. Therefore, we can laminate $R_{\theta}^{+} \hat{U}_{i}$ with $R_{\theta}^{-} \hat{U}_{i}$ in the proportion $\frac{1}{2}$ to obtain a Young measure with centre of mass

$$
\frac{1}{2}\left(R_{\theta}^{+}+R_{\theta}^{-}\right) U_{i}=\left(\begin{array}{cc}
a & b \\
c \cos \theta & d \cos \theta \\
0 & 0
\end{array}\right)
$$

which is equal to $F$ for an appropriate choice of $\theta$. For this same $F$, we can follow the proof of proposition 7.5 (i) to obtain a laminate of $Q^{+} U_{i}, Q^{-} U_{i}$, where $Q^{ \pm}=\operatorname{diag}(1, \pm 1,1)$ in the proportion $\lambda$. Thus we have constructed two distinct laminates in $\mathcal{M}(F)$.

Finally, for all other cases, we can lift the constructions in the proof of proposition 7.5 to prove non-uniqueness.

\section{Approximate relaxed energy}

The relaxation of the variational problem (1.1) is obtained by replacing $W$ with its lower quasiconvex envelope

$$
W^{\mathrm{qc}}=\sup \{\Phi: \Phi \leqslant W, \Phi \text { quasiconvex }\} .
$$

It follows from the invariance of $W$ that

$$
W^{\mathrm{qc}}(F)=W^{\mathrm{qc}}(Q F)
$$


(a)

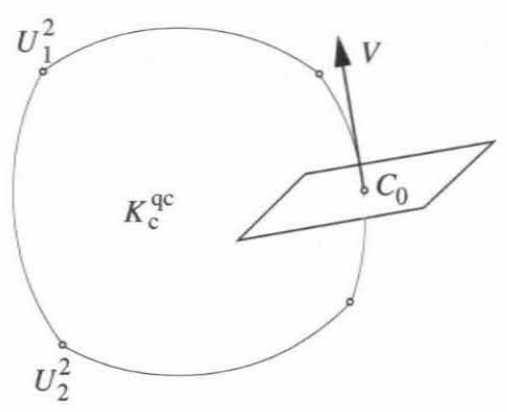

(b)

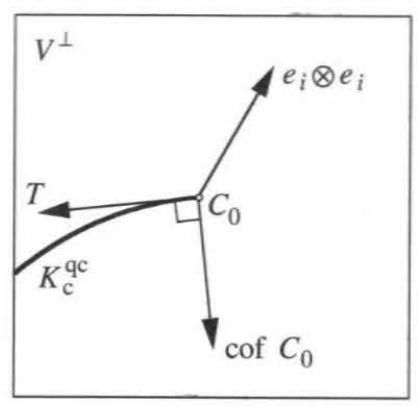

Figure 7. The quasiconvex hull and the space $V^{\perp}$.

for all $Q \in \mathrm{SO}(2), \mathrm{O}(2)$ and $\mathrm{SO}(3)$, respectively. Thus there exists $\bar{W}^{\mathrm{qc}}: \mathbb{M}_{\text {sym }}^{2 \times 2} \rightarrow \mathbb{R}$ such that

$$
W^{\mathrm{qc}}(F)=\bar{W}^{\mathrm{qc}}\left(F^{\mathrm{T}} F\right) ;
$$

the function $\bar{W}^{\mathrm{qc}}$ vanishes on $K_{\mathrm{c}}^{\mathrm{qc}}$ and grows away from it. We are interested in calculating this function, but this is extremely difficult. However, the practical interest in this function lies near the set $K_{\mathrm{c}}^{\mathrm{qc}}$. Therefore, we construct a function $\bar{W}: \mathbb{M}_{\text {sym }}^{2 \times 2} \rightarrow \mathbb{R}$, which we call the approximate relaxed energy, with the following three properties.

(P1) The function $F \mapsto \bar{W}\left(F^{\mathrm{T}} F\right)$ is quasiconvex.

(P2) The function $\bar{W}$ vanishes on $K_{\mathrm{c}}^{\mathrm{qc}}$ and hence the function $F \mapsto \bar{W}\left(F^{\mathrm{T}} F\right)$ vanishes on $K^{\mathrm{qc}}$.

(P3) The function $\bar{W}$ grows quadratically away from $K_{\mathrm{c}}^{\mathrm{qc}}$.

We note that $\bar{W}$ needs to grow quadratically in $C=F^{\mathrm{T}} F$ away from $K_{\mathrm{c}}^{\mathrm{qc}}$ in order that the 'linearized elastic moduli' are positive.

Our approximate relaxed energies are modifications of the functions $\Phi$ constructed in lemmas 3.2, 4.1 and in the proof of theorem 1.3. Recall that (H1) and $(\mathrm{H} 2)$ have been defined in $\S 6$.

REMARK 8.1. Suppose $K=\operatorname{SO}(2) U_{1} \cup \cdots \cup \operatorname{SO}(2) U_{k}$ for $U_{i} \in \mathbb{M}_{\text {sym }}^{2 \times 2}$ that satisfy (H1) and (H2), and that $\alpha, \alpha_{i}>0$. Then the function

$$
\bar{W}(C)=h(\operatorname{det} C)+\sum_{i=1}^{k} \alpha_{i}\left(\left\langle e_{i}, C e_{i}\right\rangle-m_{\mathcal{U}}\left(e_{i}\right)\right)_{+}^{2}
$$

has the properties (P1)-(P3). Here, $\mathcal{E}=\left\{e_{1}, \ldots, e_{k}\right\}$ is the set of special directions according to theorem $1.1, t_{+}^{2}=(\max \{t, 0\})^{2}$ is the square of the positive part of $t$ and $h: \mathbb{R}^{+} \rightarrow \mathbb{R}$ is a convex function that satisfies

$$
h\left(\delta^{2}\right)=h^{\prime}\left(\delta^{2}\right)=0, \quad h^{\prime \prime}\left(\delta^{2}\right)=\alpha>0 \quad \text { and } \quad h(t) \rightarrow \infty \quad \text { as } t \rightarrow 0 \text { or } \infty .
$$

The convexity of $h$ and $(\cdot)_{+}^{2}$ implies that the function $F \mapsto \bar{W}\left(F^{\mathrm{T}} F\right)$ is polyconvex and hence (P1) holds; (P2) follows from the characterization of $K^{\mathrm{qc}}$ in theorem 1.1. 
We now turn to (P3). Recall the identification of symmetric matrices with $\mathbb{R}^{3}$. In this space, the set $K_{\mathrm{c}}^{\mathrm{qc}}$ is a simply connected region in a two-dimensional manifold (det $C=\delta^{2}$ ) whose boundaries are made up of $k$ curves (the intersection of the manifold with the planes $\left.\left\langle e_{i}, C e_{i}\right\rangle=m_{\mathcal{U}}\left(e_{i}\right)\right)$. First pick any point $C_{0}$ in the interior of $K_{\mathrm{c}}^{\mathrm{qc}}$. It follows from the properties of $h$ that $\bar{W}$ grows quadratically away from $C_{0}$ in the direction perpendicular to the manifold at $C_{0}$. Now pick any point $C_{0}$ on any of the boundary curves. Let $V$ be the tangent (or velocity vector) to the curve at $C_{0}$ (see figure 7 ) and consider the plane perpendicular to $V$ (figure $7 a$ ). Since

$$
\left.\frac{\mathrm{d}}{\mathrm{d} t} \operatorname{det}\left(C_{0}+t D\right)\right|_{t=0}=\left\langle\operatorname{cof} C_{0}, D\right\rangle,
$$

the normal to the manifold at $C_{0}$ is in the direction cof $C_{0}$. Similarly, the normal to the plane is in the direction $\left(e_{i} \otimes e_{i}\right)$. Both lie on the plane $V^{\perp}$, as shown in figure $7 b$, and they are not parallel (cof $C_{0}$ has rank two, while $\left(e_{i} \otimes e_{i}\right)$ has rank one). Now, $h$ grows quadratically in the directions $\pm \operatorname{cof} C_{0}$, while $\alpha_{i}\left(\left\langle e_{i}, C e_{i}\right\rangle-m_{\mathcal{U}}\left(e_{i}\right)\right)_{+}^{2}$ grows quadratically in the direction $\left(e_{i} \otimes e_{i}\right)$. Consequently, in the plane $V^{\perp}, \bar{W}$ grows quadratically in every direction away from $T$ that is tangent to $K_{\mathrm{c}}^{\mathrm{qc}}$; in fact, given any $\varepsilon, \theta_{0}>0$, there exists $\alpha_{0}$ such that

$\bar{W}(C) \geqslant \alpha_{0}\left|C-C_{0}\right|^{2} \quad \forall C \in V^{\perp} \quad$ such that $\left|C-C_{0}\right|<\varepsilon$, angle $\left(C-C_{0}, T\right)>\theta_{0}$.

Note that, for given $\theta_{0}$ and $\varepsilon$, the constant $\alpha_{0}$ depends smoothly on $C_{0}$ and $e_{i} \otimes e_{i}$. Further, the estimate is also true even if $C_{0}$ is chosen at the intersection of two curves (i.e. if $C_{0}=U_{i}^{2}$ ); in fact, such points are obtained as the intersection of two planes $\left\langle e_{i_{1}}, C e_{i_{1}}\right\rangle=m_{\mathcal{U}}\left(e_{i_{1}}\right)$ and $\left\langle e_{i_{2}}, C e_{i_{2}}\right\rangle=m_{\mathcal{U}}\left(e_{i_{2}}\right)$ with the manifold and we may use either $e_{i_{1}}$ or $e_{i_{2}}$ to establish it. Therefore, given any $\theta_{0}, \varepsilon>0$, we can choose $\alpha_{0}$ independent of the position $C_{0}$ on boundary of $K_{\mathrm{c}}^{\mathrm{qc}}$ in the above estimate.

REMARK 8.2. Suppose $K=\mathrm{O}(2) U_{1} \cup \cdots \cup \mathrm{O}(2) U_{k}$ for $U_{i} \in \mathbb{M}_{\text {sym }}^{2 \times 2}$ that satisfy (H1), $(\mathrm{H} 2)$ or that $K=\mathrm{SO}(3) \hat{U}_{1} \cup \cdots \cup \mathrm{SO}(3) \hat{U}_{k}$ for $U_{i} \in \mathbb{M}^{3 \times 3}$ that satisfy $\left(\mathrm{H} 1^{\prime}\right),\left(\mathrm{H}^{\prime}\right)$. Then the function

$$
\bar{W}(C)=\left(\operatorname{det} C-\delta^{2}\right)_{+}^{2}+\max _{e \in S^{1}}\left(\langle e, C e\rangle-m_{\mathcal{U}}(e)\right)_{+}^{2}
$$

has the properties (P1)-(P3). This is quite similar to the discussion above.

Unfortunately, the formula (8.2) above is unsatisfactory, since it is not explicit. However, it is possible to make it explicit for specific examples.

EXAMPLE 8.3 (The four-well problem). Consider the four-well problem described in example 4.5. Given any $C \in \mathbb{M}_{\text {sym }}^{2 \times 2}$, let

$$
D= \begin{cases}C-U_{1}^{2}, & C_{11}-C_{22} \geqslant 0 \text { and } C_{12} \geqslant 0, \\ C-U_{2}^{2}, & C_{11}-C_{22} \leqslant 0 \text { and } C_{12} \geqslant 0, \\ C-U_{4}^{2}, & C_{11}-C_{22} \leqslant 0 \text { and } C_{12} \leqslant 0, \\ C-U_{3}^{2}, & C_{11}-C_{22} \geqslant 0 \text { and } C_{12} \leqslant 0 .\end{cases}
$$


Then $\bar{W}$ defined in (8.2) can be explicitly written as

$$
\bar{W}(C)=\left(\operatorname{det} C-\delta^{2}\right)_{+}^{2}+\left\{\begin{array}{lr}
(\lambda(D))_{+}^{2}, & \left(C_{11}-C_{22}\right)\left(D_{11}-D_{22}\right) \geqslant 0 \\
\max _{e \in \mathcal{E}_{4}}(\langle e, D e\rangle)_{+}^{2} & \text { and } D_{12} C_{12} \geqslant 0
\end{array}\right.
$$

where

$$
\lambda(D)=\frac{1}{2}\left(D_{11}+D_{22}\right)+\sqrt{\left(\frac{1}{2}\left(D_{11}+D_{22}\right)\right)^{2}+D_{12}^{2}}
$$

and

$$
\mathcal{E}_{4}=\left\{\frac{1}{\sqrt{ } 2}\left(\begin{array}{l}
1 \\
1
\end{array}\right), \frac{1}{\sqrt{ } 2}\left(\begin{array}{c}
1 \\
-1
\end{array}\right),\left(\begin{array}{l}
1 \\
0
\end{array}\right),\left(\begin{array}{l}
0 \\
1
\end{array}\right)\right\} .
$$

We note that

$$
\max _{e \in S^{1}}\left(\langle e, C e\rangle-m_{\mathcal{U}}(e)\right)_{+}^{2}=\left(\max _{e \in S^{1}}\left(\langle e, C e\rangle-m_{\mathcal{U}}(e)\right)\right)_{+}^{2},
$$

and hence our task is to calculate

$$
\max _{e \in S^{1}}\left(\langle e, C e\rangle-m_{\mathcal{U}}(e)\right) .
$$

Let $e=\{\cos \theta, \sin \theta\}$ and

$$
f(\theta)=\langle e, C e\rangle-m_{\mathcal{U}}(e) .
$$

It is sufficient to look at this function for $\theta \in[0, \pi)$, since our original function is invariant under $e \mapsto-e$. Our task is now to calculate $\max f$ for $\theta \in[0, \pi)$.

But first, we have to calculate $m_{\mathcal{U}}(e)=\max _{i=1, \ldots, 4}\left\langle e, U_{i}^{2} e\right\rangle$. For any $A \in \mathbb{M}_{\text {sym }}^{2 \times 2}$,

$$
\langle e, A e\rangle=A_{11} \cos ^{2} \theta+2 A_{12} \cos \theta \sin \theta+A_{22} \sin ^{2} \theta,
$$

and it is easy to conclude that

$$
m_{\mathcal{U}}(e)=\max _{i=1, \ldots, 4}\left\langle e, U_{i}^{2} e\right\rangle= \begin{cases}\left\langle e, U_{1}^{2} e\right\rangle, & \theta \in\left[0, \frac{1}{4} \pi\right], \\ \left\langle e, U_{2}^{2} e\right\rangle, & \theta \in\left[\frac{1}{4} \pi, \frac{1}{2} \pi\right], \\ \left\langle e, U_{3}^{2} e\right\rangle, & \theta \in\left[\frac{1}{2} \pi, \frac{3}{4} \pi\right], \\ \left\langle e, U_{4}^{2} e\right\rangle, & \theta \in\left[\frac{3}{4} \pi, \pi\right],\end{cases}
$$

since $a>b, c>0$ by assumption.

We now claim that $\langle e, C e\rangle$ and $f(\theta)$ achieve their maximum in the same 'quarter interval' $\left[0, \frac{1}{4} \pi\right],\left[\frac{1}{4} \pi, \frac{1}{2} \pi\right],\left[\frac{1}{2} \pi, \frac{3}{4} \pi\right]$ or $\left[\frac{3}{4} \pi, \pi\right]$. This is easily verified by contradiction. Let us consider the case $C_{22} \geqslant C_{11}, C_{12} \geqslant 0$. Then $\langle e, C e\rangle$ achieves its maximum in $\left[\frac{1}{4} \pi, \frac{1}{2} \pi\right]$. First assume that $f(\theta)$ achieves its maximum for $\theta \in\left[\frac{3}{4} \pi, \pi\right]$. Let $\varphi=\theta-\frac{1}{2} \pi$, so that $\varphi \in\left[\frac{1}{4} \pi, \frac{1}{2} \pi\right]$. Then a simple calculation using (8.4) shows that

$$
f(\varphi)-f(\theta)=\left(C_{22}-C_{11}\right)\left(\cos ^{2} \theta-\sin ^{2} \theta\right)-4 C_{12} \cos \theta \sin \theta \geqslant 0,
$$

which contradicts the assumption that $f$ achieves its maximum at $\theta$. Similarly, we can show that $f(\theta)$ cannot achieve its maximum for $\theta \in\left[0, \frac{1}{4} \pi\right]$ or for $\theta \in\left[\frac{1}{2} \pi, \frac{3}{4} \pi\right]$ by checking with $\varphi=\frac{1}{2} \pi-\theta$ and $\varphi=\pi-\theta$, respectively. We can treat the other cases similarly. 
Thus the maximum of $f$ is equal to the maximum of $\langle e, D e\rangle$ (for $D$ defined above) for $\theta$ restricted to the quarter interval in which $\langle e, C e\rangle$ achieves its maximum. Now, if the angle corresponding to the eigenvector of the maximal eigenvalue of $D$ lies in this interval, then the maximum of $\langle e, D e\rangle$ and that of $f$ is equal to the maximum eigenvalue of $D$. This is the first possibility in (8.3). If the corresponding angle lies outside this interval, then the maximum of $f$ is equal to the larger of the values of $\langle e, D e\rangle$ at the two boundaries of the interval. This is the other possibility of (8.3).

\section{Acknowledgments}

This work was conducted while K.B. visited the MPI Leipzig in 1997, 1998 and while G.D. held a position at the Max-Planck-Institute for Mathematics in the Sciences, Leipzig, and also visited Caltech in 1998. We gratefully acknowledge the partial financial support of the NSF (CMS 9457573) and AFOSR/MURI (F 4960298-1-0433).

\section{References}

1 J. M. Ball. A version of the fundamental theorem of Young measures. In PDEs and continuum models of phase transitions (ed. M. Rascle, D. Serre and M. Slemrod), Lecture Notes in Physics, vol. 344, pp. 207-215 (Springer, 1989).

2 J. M. Ball and R. D. James. Fine phase mixtures as minimizers of energy. Arch. Ration. Mech. Analysis 100 (1987), 13-52.

3 J. M. Ball and R. D. James. A characterization of plane strain. Proc. R. Soc. Lond. A 432 (1991), 93-99.

4 J. M. Ball and R. D. James. Proposed experimental tests of a theory of fine microstructure and the two-well problem. Phil. Trans. R. Soc. Lond. A 338 (1992), 389-450.

5 J. M. Ball, B. Kirchheim and J. Kristensen. Regularity of quasiconvex envelopes. Calc. Var. Partial Diff. 11 (2000), 333-359.

6 K. Bhattacharya and G. Dolzmann. Relaxed constitutive relations for phase-transforming materials. J. Mech. Phys. Solids 48 (2000), 1493-1517.

7 K. Bhattacharya and R. D. James. A theory of thin films of martensitic materials with applications to microactuators. J. Mech. Phys. Solids 47 (1999), 531-576.

8 M. Chipot and D. Kinderlehrer. Equilibrium configurations of crystals. Arch. Ration. Mech. Analysis 103 (1988), 237-277.

9 B. Dacorogna. Direct methods in the calculus of variations (Springer, 1989).

10 B. Dacorogna and P. Marcellini. Théorèmes d'existence dans les cas scalaire et vectoriel pour les équations de Hamilton-Jacobi. C. R. Acad. Sci. Paris 322 (1996), 237-240.

11 B. Dacorogna and P. Marcellini. Sur le problème de Cauchy-Dirichlet pour les systèmes d'équations non linéaires du premier ordre. C. R. Acad. Sci. Paris 323 (1996), 599-602.

12 J. L. Ericksen. On the kinematic conditions of compatibility. J. Elasticity 26 (1991), 65-74.

13 M. Gromov. Partial differential relations (Springer, 1986).

14 D. Kinderlehrer and P. Pedregal. Characterization of Young measures generated by gradients. Arch. Ration. Mech. Analysis 115 (1991), 329-365.

15 H. Le Drét and A. Raoult. The nonlinear membrane model as a variational limit of nonlinear three-dimensional elasticity. J. Math. Pure. Appl. 74 (1995), 549-578.

16 S. Müller and V. Šverák. Attainment results for the two-well problem by convex integration. In Geometric analysis and the calculus of variations (ed. J. Jost), pp. 239-251 (International Press, 1996).

17 S. Müller and V. Šverák. Convex integration with constraints and applications to phase transitions and partial differential equations. J. Eur. Math. Soc. 1 (1999), 393-422.

18 P. Pedregal. Laminates and microstructure. Eur. J. Appl. Math. 4 (1993), 121-149.

19 V. Šverák. Lower semicontinuity of variational integrals and compensated compactness. In Proc. Int. Cong. Math. 1994 (ed. S. D. Chatterji), vol. 2, pp. 1153-1158 (Basel: Birkhäuser, 1995). 
20 L. Tartar. Compensated compactness and partial differential equations. In Nonlinear analysis and mechanics: Heriot-Watt Symposium (ed. R. Knops), vol. IV, pp. 136-212 (Boston, MA: Pitman, 1979).

(Issued 27 April 2001) 